\title{
High resolution spectroscopy of the hot post-AGB stars IRAS 13266-5551 (CPD-55 5588) and IRAS 17311-4924 (Hen3-1428) ${ }^{\star \star \star \star}$
}

\author{
G. Sarkar ${ }^{1, \star \star \star}$, M. Parthasarathy ${ }^{2}$, and B. E. Reddy ${ }^{2, \dagger}$ \\ ${ }^{1}$ Inter-University Centre for Astronomy and Astrophysics, Post Bag 4, Ganeshkhind, Pune 411007, India \\ e-mail: gsarkar@iitk.ac.in \\ 2 Indian Institute of Astrophysics, Koramangala, Bangalore 560034, India
}

Received 22 June 2004 / Accepted 23 September 2004

\begin{abstract}
High resolution spectra covering the wavelength range $4900 \AA$ to $8250 \AA$ of the hot post-AGB stars IRAS 13266-5551 (CPD-55 5588) and IRAS 17311-4924 (Hen3-1428) reveal absorption lines of C II, N II, O II, Al III, Si III and Fe III and a rich emission line spectrum consisting of H I, He I, C II, N I, O I, Mg II, Al II, Si II, V I, Mn I, Fe III, [Fe II] and $[\mathrm{Cr}$ II]. The presence of [N II] and [O I] lines and absence of [O III] indicate low excitation nebulae around these stars. The components of $\mathrm{Na}$ I absorption lines indicate the presence of neutral circumstellar envelopes in addition to the low excitation nebulae around these two hot post-AGB stars. The $\mathrm{H}_{\alpha}$ lines show P-Cygni profiles indicating ongoing post-AGB mass loss. From the absorption lines we derived heliocentric radial velocities of $65.31 \pm 0.34 \mathrm{~km} \mathrm{~s}^{-1}$ and $27.55 \pm 0.74 \mathrm{~km} \mathrm{~s}^{-1}$ for IRAS 13266-5551 and IRAS 17311-4924 respectively. The high Galactic latitude and large radial velocity of IRAS 13266-5551 indicate that it belongs to the old disk population. Preliminary estimates for the CNO abundances in IRAS 13266-5551 are obtained.
\end{abstract}

Key words. stars: AGB and post-AGB - stars: early-type - stars: abundances - stars: evolution

\section{Introduction}

From the study of IRAS sources with far-IR colours similar to those of planetary nebulae (PNe) several cool and hot postAGB stars have been discovered (Parthasarathy \& Pottasch 1986; Parthasarathy et al. 2000a, 2001) forming an evolutionary sequence in the transition region from the tip of the AGB to the early stages of PNe (Parthasarathy 1993a,b). IRAS 13266-5551 (CPD-55 5588) and IRAS 17311-4924 (Hen3-1428) were identified as hot post-AGB stars (Table 1) based on their far-IR flux distribution, high Galactic latitudes and B-supergiant spectra in the optical (Parthasarathy \& Pottasch 1989; Parthasarathy 1993a; Parthasarathy et al. 2000a). The UV (IUE) spectra of these stars show C II (1335 ̊̊), Si IV (1394 ̊, $1403 \AA$ A), C IV (1550 ̊̊)

\footnotetext{
* Based on observations made with the Victor M. Blanco $4 \mathrm{~m}$ telescope of the Cerro Tololo Inter-American Observatory, Chile.

$\star \star$ Appendices and Tables 2a, 2b, 3a and $3 \mathrm{~b}$ are only available in electronic form at http://www. edpsciences.org

$\star \star \star$ Present address: Indian Institute of Technology, Kanpur, 208016, India.

$\dagger$ Visiting Observer, Cerro Tololo Inter-American Observatory, which is operated by the Association of Universities for Research in Astronomy Inc., under contract with the US National Science Foundation.
}

and N IV (1718 ̊) lines typical of the central stars of PNe. The C IV (1550 $\AA$ ) resonance lines are blueshifted indicating stellar wind velocities of $-1821 \mathrm{~km} \mathrm{~s}^{-1}$ (CPD-55 5588) and $-1066 \mathrm{~km} \mathrm{~s}^{-1}$ (Hen3-1428) respectively (Gauba \& Parthasarathy 2003). The "30 $\mu$ feature", SiC emission at $11.5 \mu$, and UIR band at $7.7 \mu$ were detected in the ISO spectrum of IRAS 17311-4924 (Gauba \& Parthasarathy 2004). These features have been detected in the circumstellar dust shells of carbon-rich AGB stars (C-stars), post-AGB stars, proto-planetary nebulae (PPNe) and planetary nebulae (PNe) (see e.g. Hony et al. 2002; Hrivnak et al. 2000; Volk et al. 2000, 2002). Loup et al. (1990) detected CO emission in IRAS 17311-4924 typical of circumstellar shells around evolved objects.

High resolution optical spectra of only a few hot post-AGB stars have been analysed. These include IRAS 01005+7910 (Klochkova et al. 2002), IRAS 18062+2410 (SAO85766, Parthasarathy et al. 2000b; Arkhipova et al. 2001a; Mooney et al. 2002; Ryans et al. 2003), IRAS 19590-1249 (LSIV-12 ${ }^{\circ} 111$, McCausland et al. 1992; Conlon et al. 1993a; Ryans et al. 2003) and IRAS 20462+3416 (LSII+34²6, Parthasarathy 1993b; García-Lario et al. 1997; Arkhipova et al. 2001b). The optical spectra of these stars show absorption lines due to C II, N II, O II, Si II, Si III, Fe III etc. Emission lines of He I, Fe I, II and III, N I, Ni I, O I have also been 
Table 1. Details of the stars.

\begin{tabular}{ccccccccc|ccccc}
\hline \hline IRAS & Name & RA & Dec & $l$ & $b$ & Sp.Type & $V$ & $B-V$ & \multicolumn{3}{c}{ IRAS fluxes (Jy.) } \\
& & $(2000)$ & $(2000)$ & & & Optical & & & $12 \mu$ & $25 \mu$ & $60 \mu$ & $100 \mu$ \\
\hline $13266-5551$ & CPD-55 5588 & $13: 29: 50.8$ & $-56: 06: 53$ & 308.30 & +6.36 & B1Ibe & $10.68^{a}$ & $0.31^{a}$ & 0.76 & 35.90 & 35.43 & 11.66 \\
$17311-4924$ & Hen3-1428 & $17: 35: 02.49$ & $-49: 26: 26.4$ & 341.41 & -9.04 & B1IIe & $10.68^{b}$ & $0.40^{b}$ & 18.34 & 150.70 & 58.74 & 17.78 \\
\hline
\end{tabular}

Photometry is from: ${ }^{a}$ Reed (1998) and ${ }^{b}$ Kozok (1985).

Spectral types are from Parthasarathy et al. (2000a).

detected. Nebular emission lines of [O II], [N II], [S II] etc., detached cold circumstellar dust shells, OB-supergiant spectral types, high Galactic latitudes and chemical composition indicate that these are PPNe (Parthasarathy et al. 1993, 1995, 2000b). IRAS 13266-5551 and IRAS 17311-4924 are found to be similar to the objects mentioned above. In this paper we report an analysis of their high resolution spectra.

\section{Observations}

High resolution $(R \sim 30000)$ spectra of IRAS 13266-5551 and IRAS 17311-4924 from $4900 \AA$ to $8250 \AA$ were obtained on 22nd June, 2002. Each object was observed twice during the night. The echelle spectrograph at the f/7.8 Ritchey-Chrétien focus of the Victor M. Blanco $4 \mathrm{~m}$ telescope of the Cerro Tololo Inter-American Observatory (CTIO), Chile was used for the purpose. The spectra were recorded using a Tektronix $2048 \times$ 2048 CCD. The slit width was $150 \mu$ corresponding to $1^{\prime \prime}$ on the sky. An appropriate number of bias frames and flat fields were observed. A Th-Ar comparison lamp was used for wavelength calibration.

As these are hot stars, spectra in the blue would contain more absorption lines. However, our observing program and the spectrograph setup did not allow us to go shortward of $4900 \AA$. Therefore, the analysis reported in this paper is based on the spectra covering the wavelength range $4900 \AA$ to $8250 \AA$.

\section{Analysis}

The spectra were processed using standard IRAF routines. They were corrected using data in the overscan region of the CCD chip. The other reduction steps included trimming, bias subtraction, flat field correction, correction for scattered light and wavelength calibration. The two sets of reduced spectra for each object were then combined to increase the signal-to-noise $(S / N)$ ratio. The final $S / N$ ratios for IRAS 13266-5551 and IRAS 17311-4924 were estimated to be $\sim 120$. The reduced spectra were continuum-normalised and the equivalent widths $\left(W_{\lambda}\right)$ of the absorption and emission lines were measured. Whenever required, deblending was done to obtain gaussian fits to the blended line profiles. The continuum-normalised spectra are presented in Appendices A and B (Figs. A and B, available at http://www.edpsciences.org). The line identifications (Tables 2a-d, 3a-d) are based on the Moore multiplet table (1945) and the linelists of Parthasarathy et al. (2000b) and Klochkova et al. (2002). Unidentified lines are denoted by "UN". Night sky emission lines (atmospheric emission) were identified from Osterbrock \& Martel (1992) and Osterbrock et al. (1996) and are listed as "atmos." in the tables. The laboratory wavelengths, $\log (g f)$ values and excitation potentials $(\chi)$ are from the linelist compiled by Ivan Hubeny and retrieved from the directory /pub/hubeny/synplot by anonymous ftp from tlusty.gsfc.nasa.gov.

\subsection{Description of the spectra}

The high resolution optical spectra of IRAS 13266-5551 and IRAS 17311-4924 show absorption lines due to C II, N II, O II, $\mathrm{Ne} \mathrm{I,} \mathrm{Al} \mathrm{III} \mathrm{and} \mathrm{Si} \mathrm{III.} \mathrm{The} \mathrm{O} \mathrm{I} \mathrm{triplet} \mathrm{at} \sim 7773 \AA$ was detected in both stars. Both stars show a rich emission line spectrum with lines of C II, Mg II, Al II, Si II, Fe III and [Cr II] in emission. Emission lines of N I, O I, [O I], V I, Mn I and [Fe II] in IRAS 17311-4924 were also detected. The presence of low excitation nebular lines of [N II] in the spectra of both stars and the absence of [O III] $5007 \AA$ indicate that photoionisation has just started.

The He I lines in the two stars show a variety of profiles. They appear in absorption, in emission and also show P-Cygni profiles indicating post-AGB mass-loss. The He I(4) 5015.678 $\mathrm{A}$ and He I(45) $7281.349 \AA$ emission lines in IRAS 13266-5551 are superposed on the corresponding absorption components. The asymmetric nature of these emission lines suggests that they may have P-Cygni profiles. The presence of high excitation lines of He I and low excitation emission lines of Na I (see Sect. 3.5) and V I indicate a range of temperatures for the circumstellar material around these stars. The circumstellar envelope around these stars may be extended and the outermost regions may be cooler. The $\mathrm{H}_{\alpha}$ lines in both stars show P-Cygni profiles. The emission peak of the line in IRAS 17311-4924 is asymmetric.

\subsection{Radial velocities}

Heliocentric radial velocities $\left(V_{\mathrm{r}}\right)$ for the well defined absorption and emission lines are presented in Tables $2 \mathrm{a}, 2 \mathrm{~b}, 2 \mathrm{~d}, 3 \mathrm{a}$, $3 \mathrm{~b}$ and $3 \mathrm{~d}$. The radial velocities of the Fe III (5) absorption lines in IRAS 13266-5551 are relatively larger than the rest and those of the Ne I absorption lines in IRAS 17311-4924 are relatively smaller suggesting that these lines may be formed in different regions in the atmospheres of these stars. Therefore, in estimating the mean heliocentric radial velocities, we have excluded the above lines. The radial velocity of 
Table 2c. Lines with P-Cygni profiles in IRAS 13266-5551 (CPD-55 5588). Equivalent widths of the absorption and emission components of the P-Cygni profiles are given. Wind velocities are estimated from the blue absorption edges of the P-Cygni profiles.

\begin{tabular}{ccccccc}
\hline \hline $\begin{array}{c}\lambda_{\text {lab }} \\
(\AA)\end{array}$ & Ident. & $\begin{array}{c}\mathrm{W}_{\lambda} \text { (absorption) } \\
(\AA)\end{array}$ & $\begin{array}{c}W_{\lambda} \text { (emission) } \\
(\AA)\end{array}$ & $\log (g f)$ & $\begin{array}{c}\chi \\
(\mathrm{eV})\end{array}$ & $\begin{array}{c}\text { Wind Velocity } \\
\mathrm{km} \mathrm{s}^{-1}\end{array}$ \\
\hline 5875.618 & He I (11) & 0.079 & 0.484 & 0.410 & $20.97-23.08$ & \\
+5875.650 & He I (11) & & & -0.140 & $20.97-23.08$ \\
+5875.989 & He I (11) & & & -0.210 & $20.97-23.08$ & \\
6562.817 & $\mathrm{H}_{\alpha}$ & & 6.902 & 0.710 & $10.15-12.04$ & \\
$6582.882^{\dagger}$ & $\mathrm{C} \mathrm{II} \mathrm{(2)}$ & 0.031 & & -0.180 & $14.45-16.33$ & \\
6678.149 & He I (46) & 0.212 & 0.076 & 0.330 & $21.22-23.08$ & -101.34 \\
\hline
\end{tabular}

Owing to the broad wings of the $\mathrm{H}_{\alpha}$ emission component, the absorption component of the P-Cygni profile lies above the normalised continuum (see Fig. 3). ${ }^{\dagger}$ The emission component of C II(2) 6582.882 Å P-Cygni profile is blended with [N II](1F) $6583.6 \AA$.

Table 2d. Absorption (a) and emission (e) components of Na I $D_{2}\left(5889.953 \AA\right.$ ) and Na $\mathrm{I}_{1}(5895.923 \AA$ ) lines in the spectrum of IRAS 13266-5551 (CPD-55 5588). $W_{\lambda}$ are the equivalent widths of the components and $V_{\mathrm{r}}$ are the respective heliocentric radial velocities.

\begin{tabular}{c|c|ccc|ccc}
\hline \hline \multirow{3}{*}{ Component } & & \multicolumn{7}{|c}{ IRAS 13266-5551 } \\
\cline { 3 - 8 } & & \multicolumn{5}{|c}{$\mathrm{Na} \mathrm{I} \mathrm{D}_{2}$} \\
\cline { 3 - 8 } & & $\begin{array}{c}\lambda_{\text {obs. }} \\
(\AA)\end{array}$ & $\begin{array}{c}W_{\lambda} \\
(\AA)\end{array}$ & $\begin{array}{c}V_{\mathrm{r}} \\
\left(\mathrm{km} \mathrm{s}^{-1}\right)\end{array}$ & $\begin{array}{c}\lambda_{\text {obs. }} \\
(\AA)\end{array}$ & $\begin{array}{c}W_{\lambda} \\
(\AA)\end{array}$ & $\begin{array}{c}V_{\mathrm{r}} \\
\left(\mathrm{km} \mathrm{s}^{-1}\right)\end{array}$ \\
\hline 1. & $\mathrm{a}$ & 5889.54 & 0.168 & -36.28 & 5895.432 & 0.062 & -40.22 \\
2. & $\mathrm{a}$ & 5890.003 & 0.551 & -12.69 & 5896.002 & 0.415 & -11.22 \\
3. & $\mathrm{a}$ & 5890.366 & 0.292 & +5.80 & 5896.38 & 0.203 & +8.01 \\
4. & $\mathrm{a}$ & 5890.938 & 0.021 & +34.92 & 5896.913 & 0.029 & +35.13 \\
5. & $\mathrm{e}$ & 5891.324 & 1.371 & +54.59 & 5897.373 & 0.007 & +58.54 \\
\hline
\end{tabular}

the C II (2) $6578.052 \AA$ absorption line has also been excluded (see footnote to Table 2a). We obtained mean radial velocities of $65.31 \pm 0.34 \mathrm{~km} \mathrm{~s}^{-1}$ and $27.55 \pm 0.74 \mathrm{~km} \mathrm{~s}^{-1}$ from the absorption lines in IRAS 13266-5551 and IRAS 17311-4924 respectively. The mean heliocentric radial velocities of the emission lines are $58.32 \pm 0.65 \mathrm{~km} \mathrm{~s}^{-1}$ and $32.74 \pm 0.43$ for IRAS 13266-5551 and IRAS 17311-4924 respectively. In estimating the mean radial velocity of the emission lines we have excluded the radial velocity measurements of the forbidden lines. The errors given here refer to the probable errors of estimation. Figures $1 \mathrm{a}$ and $\mathrm{b}$ show the overall radial velocity trend for the absorption and emission lines with respect to the equivalent widths $\left(W_{\lambda}\right)$ and lower excitation potentials of these lines.

The mean heliocentric radial velocity from absorption lines in the case of IRAS 17311-4924 corresponds to $V_{\mathrm{LSR}}=$ $31.13 \mathrm{~km} \mathrm{~s}^{-1}$. This value may be compared with the velocity $\left(V_{\mathrm{LSR}}=\right)$ of $36 \mathrm{~km} \mathrm{~s}^{-1}$ derived from CO observations of the star by Loup et al. (1990).

\subsection{Wind velocities from the P-Cygni profiles}

We estimated wind velocities from the well defined and unblended blue absorption edges of the P-Cygni profiles of He I, $\mathrm{C}$ II and Fe III (Tables $2 \mathrm{c}$ and $3 \mathrm{c}$ ). The absorption components of the $\mathrm{H}_{\alpha}$ P-Cygni profiles are affected by the broad wings of the $\mathrm{H}_{\alpha}$ emission components (Fig. 3) and hence could not be used to estimate the wind velocities in these stars. The absorption component of the He I(45) $7281.349 \AA$ P-Cygni profile in IRAS 17311-4924 may be affected by atmospheric absorption lines in this region. The wind velocities in IRAS 17311-4924 increase with the lower excitation potential (LEP) of the species involved.

\subsection{Diffuse interstellar bands (DIBs)}

DIBs are absorption features in the spectra of reddened stars and have their origin in the interstellar and circumstellar medium. They are typically broader than expected from the Doppler broadening of turbulent gas motions in the interstellar and circumstellar medium. A DIB at $5780.410 \AA$ A was identified in the spectra of IRAS 13266-5551 and IRAS 17311-4924. IRAS 13266-5551 also exhibited DIBs at $\lambda \lambda 5797.030 \AA$, $6195.990 \AA, 6203.060 \AA$ and $6613.630 \AA$. Their heliocentric radial velocities $\left(V_{\mathrm{r}}\right.$, Tables $2 \mathrm{a}$ and $\left.3 \mathrm{a}\right)$ rule out the possibility of circumstellar origin. From the strength of the band at $5780.410 \AA\left(W_{\lambda}=169.6 \mathrm{~m} \AA\right.$ for IRAS $13266-5551$ and $109.9 \mathrm{~m} \AA$ for IRAS 17311-4924), we estimate interstellar $E(B-V) \simeq 0.35$ and 0.20 respectively (Herbig 1993). At the Galactic latitude and longitude of these stars, using the Diffuse Infrared Background Experiment (DIRBE)/IRAS dust maps (Schlegel et al. 1998), we estimated interstellar extinction values of 0.53 and 0.22 respectively. Herbig (1993) concludes that although the DIB strengths increase linearly with $E(B-V)$, there is a real dispersion about the mean relationship. For 

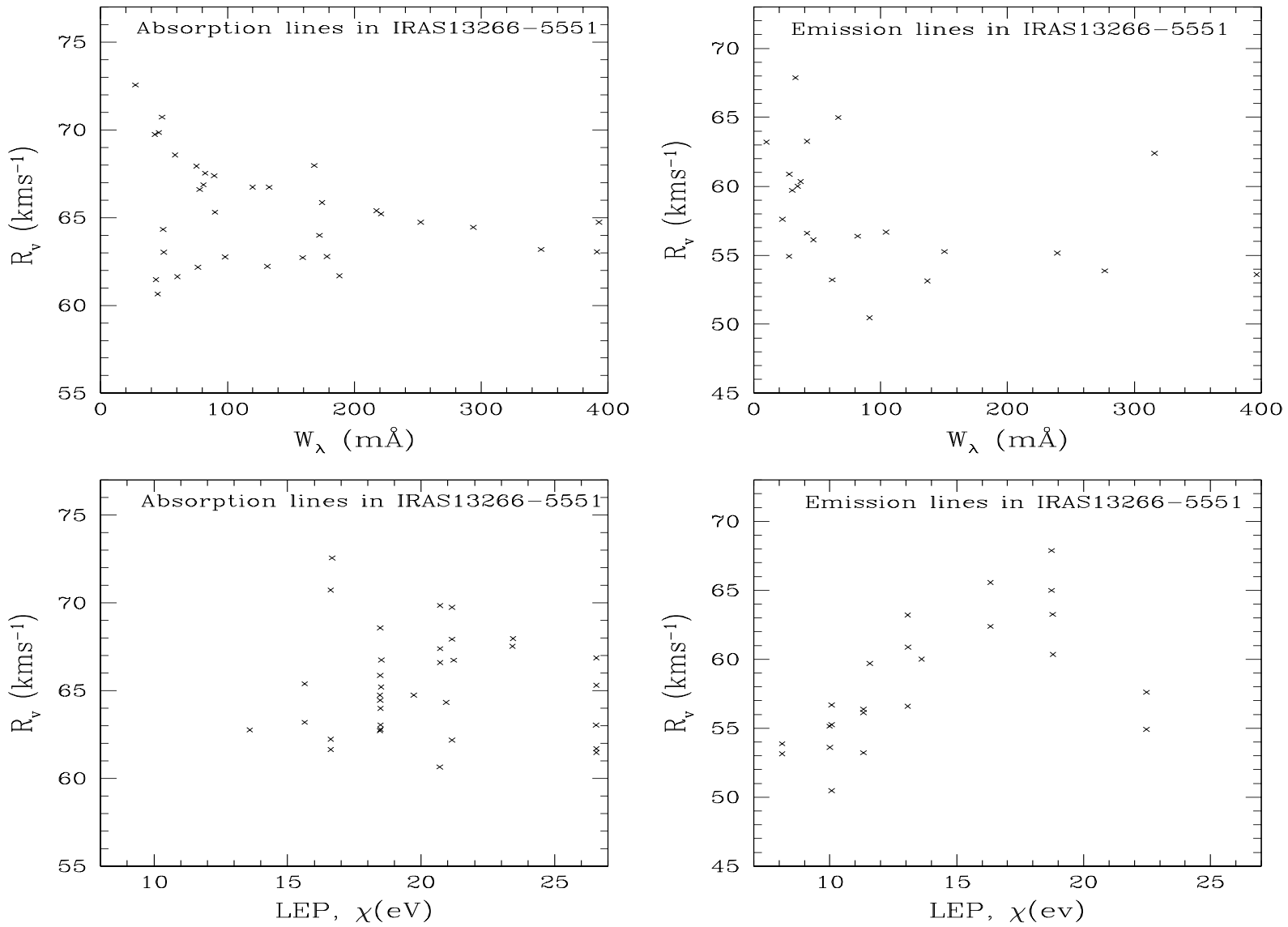

Fig. 1a. Radial velocity trends of the absorption and emission lines in IRAS 13266-5551. Radial velocity measurements of the forbidden lines have not been plotted.

Table 3c. Lines with P-Cygni profiles in IRAS 17311-4924 (Hen3-1428). Equivalent widths of the absorption and emission components of the P-Cygni profiles are given. Wind velocities are estimated from the blue absorption edges of the P-Cygni profiles.

\begin{tabular}{|c|c|c|c|c|c|c|}
\hline $\begin{array}{l}\lambda_{\text {lab }} \\
(\AA)\end{array}$ & Ident. & $\begin{array}{c}W_{\lambda} \text { (absorption) } \\
(\AA)\end{array}$ & $\begin{array}{c}W_{\lambda} \text { (emission) } \\
(\AA)\end{array}$ & $\log (g f)$ & $\begin{array}{c}\chi \\
(\mathrm{eV})\end{array}$ & $\begin{array}{c}\text { Wind velocity } \\
\mathrm{km} \mathrm{s}^{-1}\end{array}$ \\
\hline 5015.675 & He I (4) & 0.183 & 0.319 & -0.820 & $20.62-23.09$ & -146 \\
\hline 5073.78 & $\mathrm{Fe}$ III (5) & 0.015 & 0.009 & -2.557 & $8.65-11.09$ & -81.36 \\
\hline 5086.69 & Fe III (5) & 0.010 & 0.018 & -2.590 & $8.66-11.09$ & \\
\hline 5127.463 & Fe III (5) & 0.070 & 0.025 & -2.218 & $8.65-11.07$ & -81.74 \\
\hline 5156.00 & Fe III (5) & 0.037 & 0.035 & -2.018 & $8.64-11.04$ & -78.20 \\
\hline 5875.618 & He I (11) & & 0.992 & 0.410 & $20.97-23.08$ & \\
\hline+5875.650 & He I (11) & & & -0.140 & $20.97-23.08$ & \\
\hline+5875.989 & He I (11) & & & -0.210 & $20.97-23.08$ & \\
\hline 6562.817 & $\mathrm{H}_{\alpha}$ & & 9.872 & 0.710 & $10.15-12.04$ & \\
\hline 6578.03 & C II (2) & 0.327 & 0.082 & 0.120 & $14.45-16.33$ & -114.20 \\
\hline $6582.85^{\dagger}$ & C II (2) & 0.269 & & -0.180 & $14.45-16.33$ & \\
\hline 6678.149 & He I (46) & 0.417 & 0.412 & 0.330 & $21.22-23.08$ & -145.77 \\
\hline 7065.188 & He I (10) & 0.137 & 1.062 & -0.460 & $20.96-22.72$ & \\
\hline+7065.719 & He I (10) & & & -1.160 & $20.96-22.72$ & \\
\hline 7281.349 & He I (45) & 0.151 & 0.229 & -0.840 & $21.22-22.92$ & \\
\hline
\end{tabular}

Two blended absorption components are visible in the P-Cygni profile of $\mathrm{He} \mathrm{I}(11)$. The emission peak of the $\mathrm{H}_{\alpha} \mathrm{P}-\mathrm{Cygni}$ profile is asymmetric, indicating two emission components blended together. Owing to the broad wings of the emission component in $\mathrm{H}_{\alpha}$, the absorption component of the P-Cygni profile lies above the normalised continuum (see Fig. 3 ). ${ }^{\dagger}$ the emission component of the C II(2) $6582.85 \AA$ P-Cygni profile is blended with [N II] (1F) 6583.6 A. 

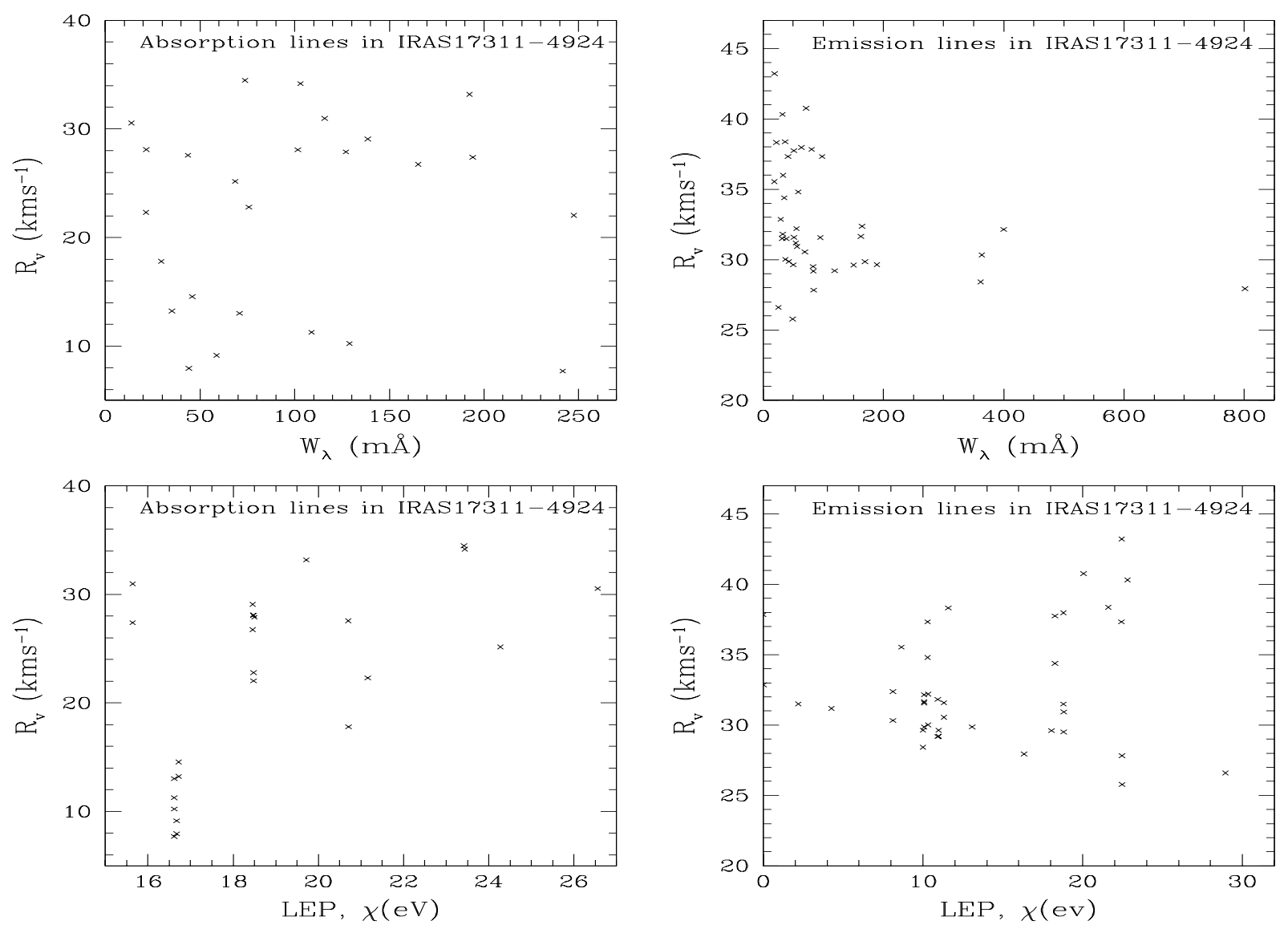

Fig. 1b. Radial velocity trends of the absorption and emission lines in IRAS 17311-4924. Radial velocity measurements of the forbidden lines have not been plotted.

Table 3d. Absorption (a) and emission (e) components of $\mathrm{Na} I \mathrm{D}_{2}\left(5889.953 \AA\right.$ ) and Na I $\mathrm{D}_{1}(5895.923 \AA)$ lines in the spectrum of IRAS 17311-4924 (Hen3-1428). $W_{\lambda}$ are the equivalent widths of the components and $V_{\mathrm{r}}$ are the respective heliocentric radial velocities.

\begin{tabular}{c|c|ccc|ccc}
\hline \hline \multirow{3}{*}{ Component } & & \multicolumn{7}{|c}{ IRAS 17311-4924 } \\
\cline { 3 - 8 } & & \multicolumn{7}{|c}{$\mathrm{Na} \mathrm{I} \mathrm{D}_{2}$} \\
\cline { 3 - 8 } & & $\begin{array}{c}\lambda_{\text {obs. }} \\
(\AA)\end{array}$ & $\begin{array}{c}W_{\lambda} \\
(\AA)\end{array}$ & $\begin{array}{c}V_{\mathrm{r}} \\
\left(\mathrm{km} \mathrm{s}^{-1}\right)\end{array}$ & $\begin{array}{c}\lambda_{\text {obs. }} \\
(\AA)\end{array}$ & $\begin{array}{c}W_{\lambda} \\
(\AA)\end{array}$ & $\begin{array}{c}V_{\mathrm{r}} \\
\left(\mathrm{km} \mathrm{s}^{-1}\right)\end{array}$ \\
\hline 1. & $\mathrm{a}$ & 5889.886 & 0.552 & -5.87 & 5895.875 & 0.479 & -4.90 \\
2. & $\mathrm{a}$ & 5890.334 & 0.121 & +16.94 & 5896.331 & 0.121 & +18.30 \\
3. & $\mathrm{e}$ & 5890.677 & 0.096 & +34.41 & 5896.695 & 0.020 & +36.82 \\
\hline
\end{tabular}

example from their data, HD144470 and HD37061 both have about the same DIB strengths despite large differences in their values of $E(B-V), 0.22$ and 0.56 respectively.

\section{5. $\mathrm{Na} I \mathrm{D}_{2}$ and $\mathrm{Na} I \mathrm{D}_{1}$ lines}

The $\mathrm{Na} I \mathrm{D}_{2}$ and $\mathrm{Na} \mathrm{I}_{1} \mathrm{D}_{1}$ lines in the spectra of IRAS 13266-5551 and IRAS 17311-4924 show both absorption and emission components (Fig. 2, Tables $2 \mathrm{~d}$ and 3d). If one compares the radial velocities of these components with the average radial velocities for each star (Sect. 3.2), it is evident that the absorption components 1,2 and 3 in IRAS 13266-5551 and component 1 in IRAS 17311-4924 are of interstellar origin. Components 4 and 2 in IRAS 13266-5551 and IRAS 17311-4924 respectively may be of circumstellar origin indicating the presence of neutral circumstellar envelopes in addition to the cold detached dust shells and low excitation nebulae.

Radial velocities of the emission components of the $\mathrm{Na}$ I lines are very close to the mean radial velocity of emission lines in IRAS 13266-5551 $\left(=+58.32 \mathrm{~km} \mathrm{~s}^{-1}\right)$ and IRAS 17311-4924 $\left(=+32.74 \mathrm{~km} \mathrm{~s}^{-1}\right)$. However, the emission components of the $\mathrm{Na}$ I lines may be disturbed by nearby absorption components and therefore the velocities derived from the $\mathrm{Na}$ I emissions may be in error.

\section{6. $H_{\alpha}$ profile and mass loss rate}

$\mathrm{H}_{\alpha}$ profiles of the two stars are shown in Fig. 3. The wavelengths were converted to velocity units relative to the 


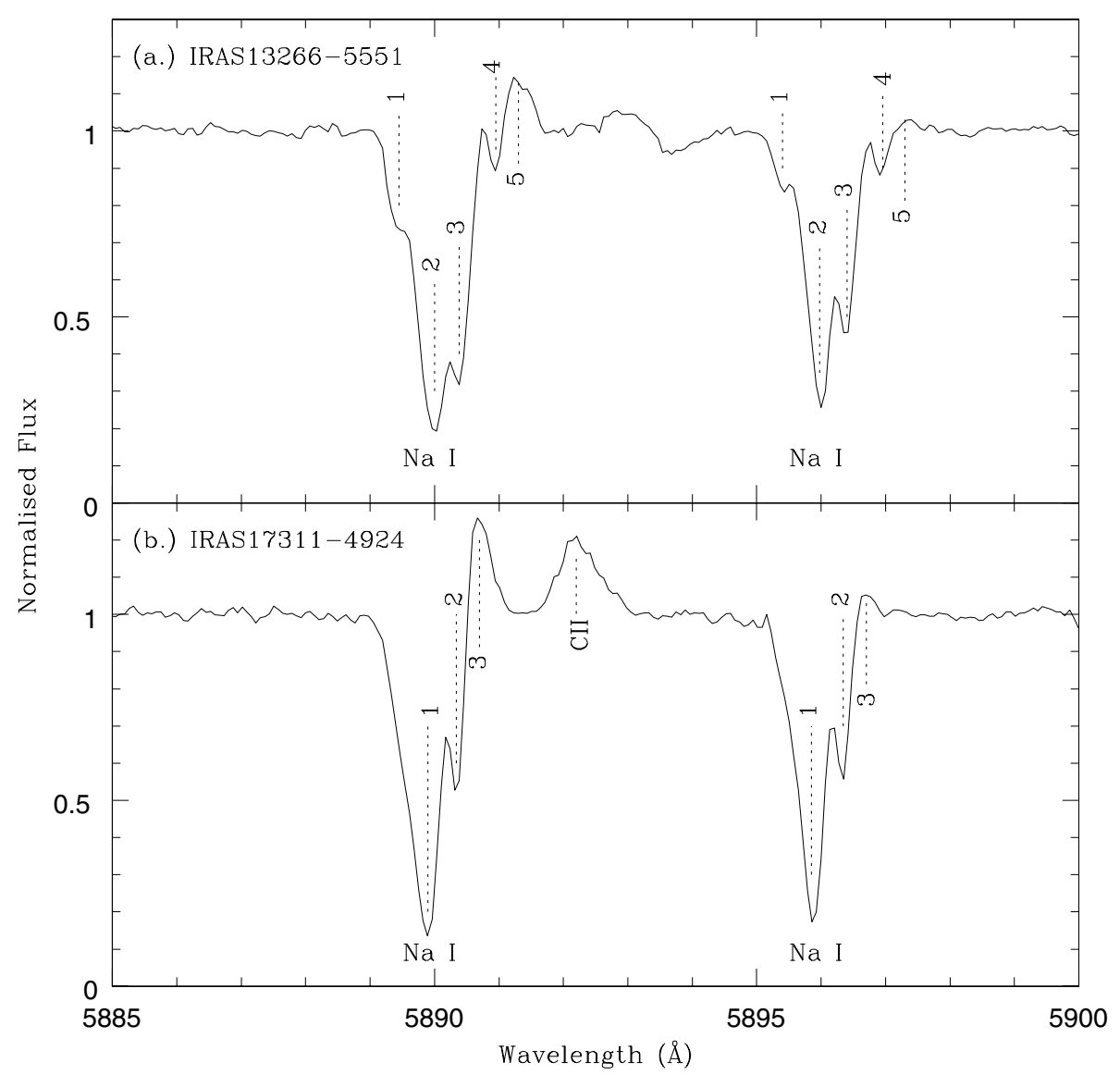

Fig. 2. $\mathrm{Na} \mathrm{I} \mathrm{D}_{2}$ and $\mathrm{Na} \mathrm{I} \mathrm{D}_{1}$ lines in the spectra of a) IRAS 13266-5551 and b) IRAS 17311-4924. The various absorption and emission components of the lines have been labelled.

laboratory wavelength of the $\mathrm{H}_{\alpha}$ line, $6562.817 \AA$ A. The zero point was then adjusted for the heliocentric radial velocity of each star. The wings of $\mathrm{H}_{\alpha}$ can be seen up to about 220 and $180 \mathrm{~km} \mathrm{~s}^{-1}$ in IRAS $13266-5551$ and IRAS $17311-4924$ respectively.

The P-Cygni profile of $\mathrm{H}_{\alpha}$ indicates ongoing mass loss. Model calculations by Klein \& Castor (1978) predicted a tight relationship between the $\mathrm{H}_{\alpha}$ luminosity of the stellar wind and mass loss rate. The $\mathrm{H}_{\alpha}$ luminosity is related to the equivalent width of the $\mathrm{H}_{\alpha}$ emission line (see e.g. Conti \& Frost 1977; Ebbets 1982). The equivalent widths of the $\mathrm{H}_{\alpha}$ emission components are $6.902 \AA$ and $9.872 \AA$ in IRAS $13266-5551$ and IRAS 17311-4924 respectively (Tables $2 \mathrm{c}$ and $3 \mathrm{c}$ ). Modelling the $\mathrm{H}_{\alpha}$ profiles to derive the mass loss rates of post-AGB stars will be the subject of a future paper. Here, we may compare our $\mathrm{H}_{\alpha}$ profiles with the B1.5Ia star, BD-14 ${ }^{\circ} 5037$. The observed equivalent width of the $\mathrm{H}_{\alpha}$ emission component in this star is 7.4 $\AA$. From the $\mathrm{H}_{\alpha}$ profile, Leitherer (1988) derived a mass loss rate of $1.58 \times 10^{-6} M_{\odot} \mathrm{yr}^{-1}$.

\subsection{Expansion velocities}

Nebular expansion velocities were estimated from the FWHM of the $[\mathrm{O} \mathrm{I}]$ and [N II] (6548.1 $\AA$ ) emission lines using $V_{\exp }=0.50 F W H M$ (Table 4). The mean expansion velocity for IRAS 17311-4924 from [O I] lines is $11.99 \mathrm{~km} \mathrm{~s}^{-1}$. The higher velocity from [N II] is due to the fact that [N II] emission dominates in the outermost ionised layers of the nebulae and $V_{\exp }$ increases with radial distance from the central stars (see e.g. Weinberger 1989).

Based on CO observations Loup et al. (1990) and Nyman et al. (1992) estimated expansion velocities of $11 \mathrm{~km} \mathrm{~s}^{-1}$ and $14.1 \mathrm{~km} \mathrm{~s}^{-1}$ respectively for IRAS $17311-4924$.

Radial velocity of absorption component 2 of the Na I profile (18.30 $\mathrm{km} \mathrm{s}^{-1}$, see Table 3d) in IRAS 17311-4924 is comparable with the expansion velocity of the star, reaffirming its possible circumstellar origin.

\subsection{Atmospheric parameters and abundances}

The presence of He I lines and the absence of He II lines in IRAS 13266-5551 and IRAS 17311-4924 indicates $18000 \mathrm{~K} \leq$ $T_{\text {eff }} \leq 25000 \mathrm{~K}$ (Miroshnichenko et al. 1998). We used Kurucz's WIDTH9 program and the spectrum synthesis code, SYNSPEC (Hubeny et al. 1985) along with solar metallicity Kurucz (1994) model atmospheres to derive the atmospheric parameters and elemental abundances under the LTE approximation.

The usual criterion for determining the effective temperature $\left(T_{\text {eff }}\right)$, gravity $(\log g)$ and microturbulent velocity $\left(\xi_{\mathrm{t}}\right)$ of a star, is to obtain zero slopes in plots of (i) $\log$ abundances for a particular species vs. lower excitation potentials of that species (ii) log abundances for two species of a particular 
IRAS $13266-5551$

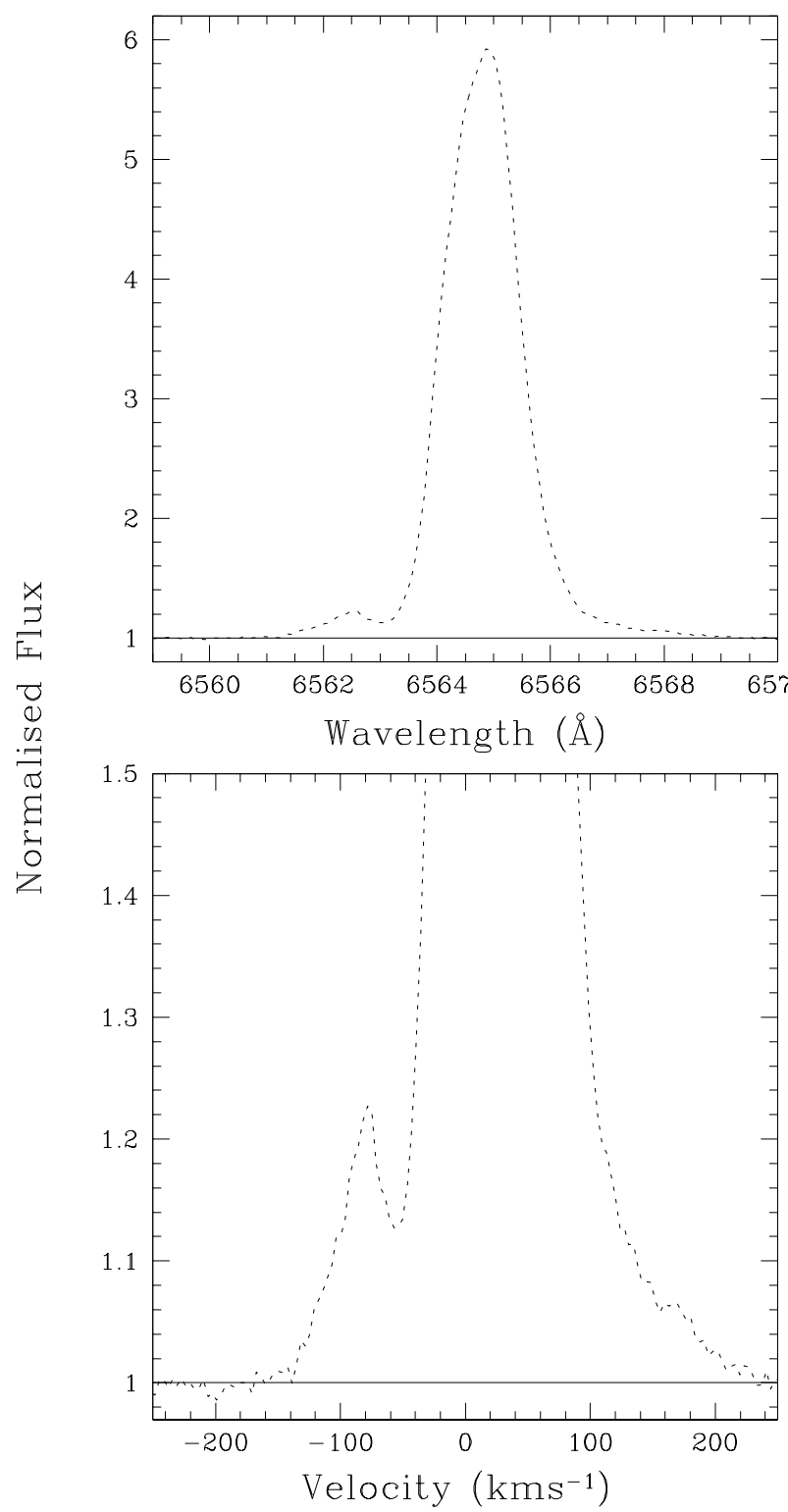

IRAS $17311-4924$

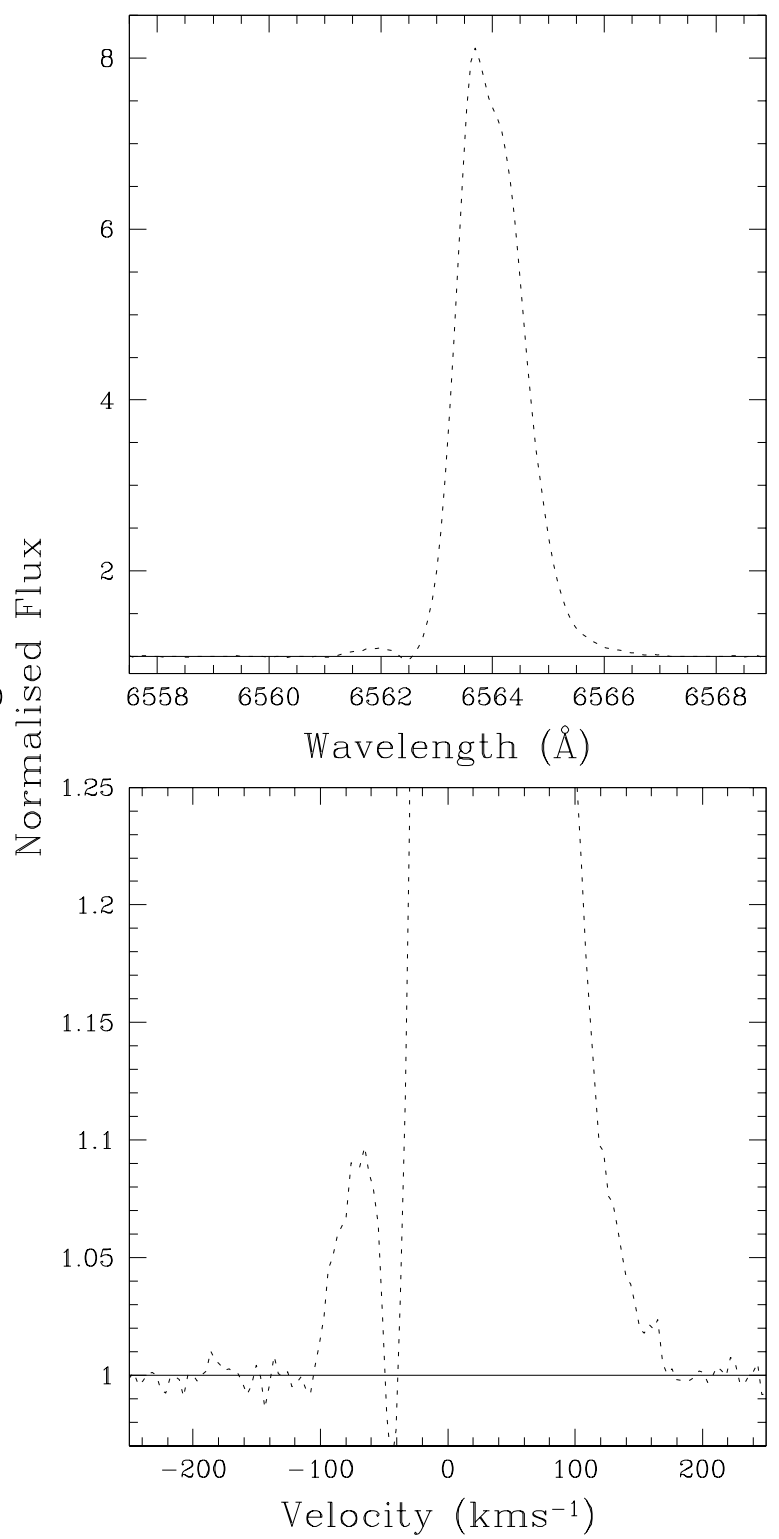

Fig. 3. The normalised $\mathrm{H}_{\alpha}$ profiles (dotted lines) of IRAS 13266-5551 and IRAS 17311-4924.

Table 4. Expansion velocities.

\begin{tabular}{cccc|cccc}
\hline \hline \multirow{3}{*}{ Ident. } & \multicolumn{2}{c|}{ IRAS 13266-5551 } & & \multicolumn{3}{c}{ IRAS 17311-4924 } \\
& $\lambda_{\text {lab }}$ & $\begin{array}{c}F W H M \\
\AA\end{array}$ & $\begin{array}{c}V_{\exp } \\
\mathrm{km} \mathrm{s}^{-1}\end{array}$ & Ident. & $\lambda_{\text {lab }}$ & $F W H M$ & $V_{\exp }$ \\
& & $\AA$ & & & $\AA$ & $\mathrm{km} \mathrm{s}^{-1}$ \\
\hline$[\mathrm{N} \mathrm{II}](1 \mathrm{~F})$ & 6548.1 & 0.845 & 19.36 & {$[\mathrm{O} \mathrm{I}](3 \mathrm{~F})$} & 5577.35 & 0.418 & 11.24 \\
& & & & {$[\mathrm{O} \mathrm{I}](1 \mathrm{~F})$} & 6300.304 & 0.527 & 12.55 \\
& & & & {$[\mathrm{O} \mathrm{I}](1 \mathrm{~F})$} & 6363.776 & 0.517 & 12.19 \\
& & & & {$[\mathrm{~N} \mathrm{II}](1 \mathrm{~F})$} & 6548.1 & 1.024 & 23.46 \\
\hline
\end{tabular}

From CO observations Loup et al. (1990) and Nyman et al. (1992) estimated expansion velocities of $11 \mathrm{~km} \mathrm{~s}^{-1}$ and $14.1 \mathrm{~km} \mathrm{~s}^{-1}$ respectively in IRAS 17311-4924. The absorption component 2 of the Na I profile in IRAS $17311-4924$ has a radial velocity $\left(=18.30 \mathrm{~km} \mathrm{~s}^{-1}\right)$ comparable with the expansion velocity of the star.

element (e.g. Fe II and Fe III) vs. lower excitation potentials and (iii) $\log$ abundances for a particular species vs. equivalent widths.
The largest number of absorption lines in the spectrum of IRAS 13266-5551 are those of N II. The majority of the $\mathrm{N}$ II lines are strong with $W_{\lambda} \geq 100 \mathrm{~m} \AA$. Besides, the observed 

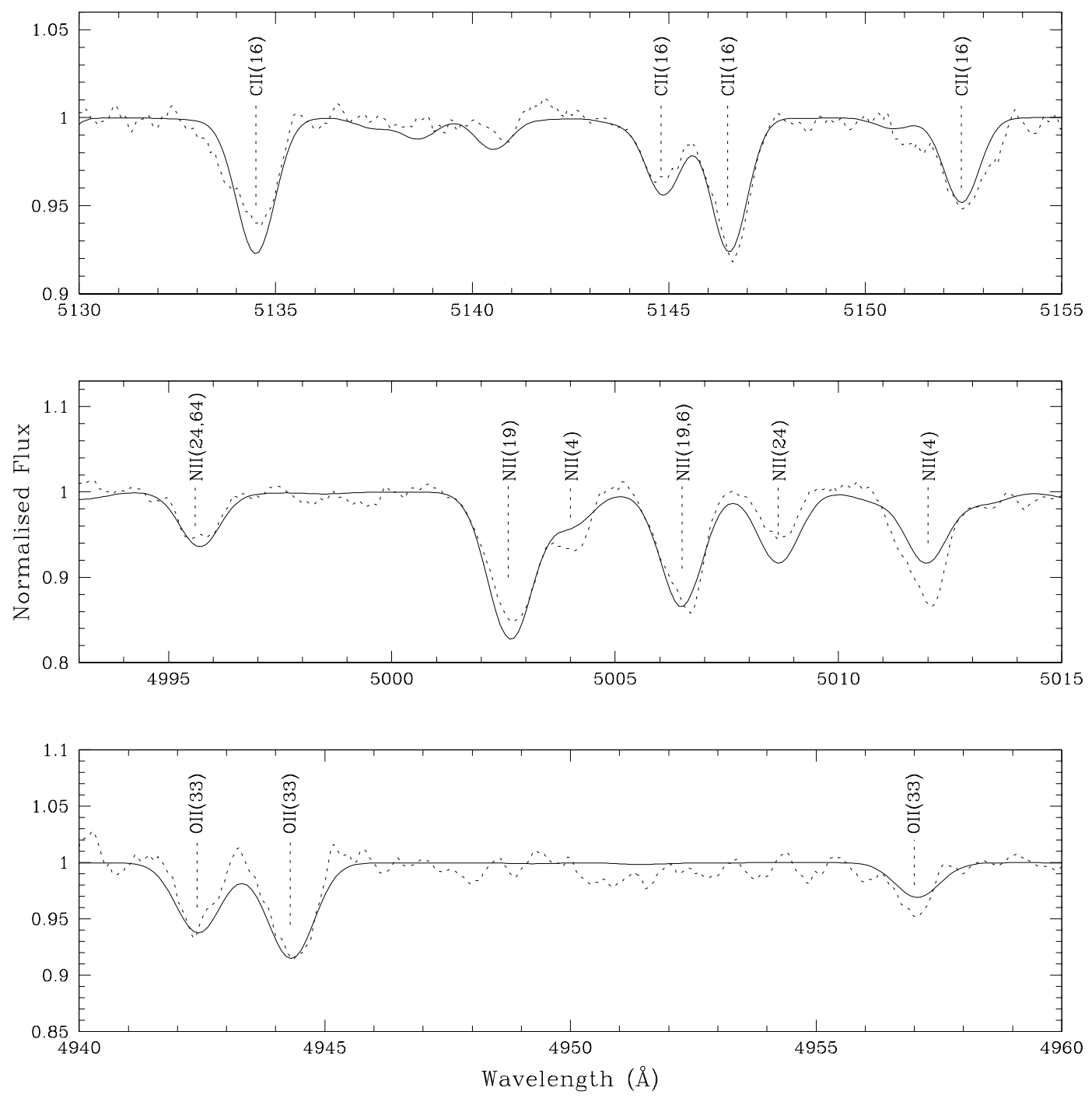

Fig. 4. Regions showing the C II, N II and O II absorption lines in the observed spectrum (dotted line) of IRAS 13266-5551 plotted along with the synthetic spectrum (solid line) for the derived atmospheric parameters $\left(T_{\text {eff }}=23000 \mathrm{~K}, \log g=3.0, \xi_{\mathrm{t}}=10 \mathrm{~km} \mathrm{~s}^{-1}\right)$ and elemental abundances (Table 5a) of the star.

$\mathrm{N}$ II lines fall in a narrow range of lower excitation potentials (Tables $2 \mathrm{a}$ and $3 \mathrm{a}$ ). This, coupled with the lack of two ionisation species of any element does not allow us to employ the usual criterion for determining $T_{\mathrm{eff}}, \log g$ and $\xi_{\mathrm{t}}$. Hence, for IRAS 13266-5551 we obtained abundances of C II, N II, O II, and Fe III with the WIDTH9 program for various combinations of $T_{\text {eff }}, \log g$ and $\xi_{\mathrm{t}}$. We covered $18000 \mathrm{~K} \leq T_{\text {eff }} \leq 24000 \mathrm{~K}$ and $5 \mathrm{~km} \mathrm{~s}^{-1} \leq \xi_{\mathrm{t}} \leq 45 \mathrm{~km} \mathrm{~s}^{-1}$. From the Kurucz (1994) model atmospheres, the $\log g$ value was limited to a minimum of 3.0. For each combination of these parameters, we then synthesised the spectrum using SYNSPEC. The best fit to the observed spectrum was obtained for $T_{\text {eff }}=23000 \mathrm{~K}, \log g=3.0, \xi_{\mathrm{t}}=$ $10 \mathrm{~km} \mathrm{~s}^{-1}$ (Fig. 4).

IRAS 17311-4924 has fewer absorption lines. The largest number (8) of absorption lines that we find in our spectrum are those of N II and Ne I. While the N II lines are strong with $W_{\lambda} \geq 100 \mathrm{~mA}$, the Ne I lines are very sensitive to NLTE effects (see Sect. 3.8.5 below). Because of the small number of C II, $\mathrm{N} \mathrm{II} \mathrm{and} \mathrm{O} \mathrm{II} \mathrm{lines} \mathrm{and} \mathrm{the} \mathrm{lack} \mathrm{of} \mathrm{iron} \mathrm{lines,} \mathrm{we} \mathrm{were} \mathrm{unable} \mathrm{to}$ estimate the atmospheric parameters and metallicity. The star suffers significant circumstellar extinction, $E(B-V)_{\text {C.S. }}=0.39$ (Gauba \& Parthasarathy 2003) and the circumstellar extinction law in the UV was found to be linear in $\lambda^{-1}$. Hence, the $(B-V)$ color of the star cannot be used to estimate the temperature. An estimate of the temperature and gravity may be made from the spectral type of the star. Parthasarathy et al. (2000b) classified it as B1IIe which corresponds to $T_{\text {eff }}=20300 \mathrm{~K}$ and $\log g=3.0$ (Lang 1992).

\subsubsection{He I lines}

We used the $5047.738 \AA \mathrm{He}$ I (47) absorption line in IRAS 13266-5551 and estimated the helium abundance in this star (Table 5a). The estimated abundance is somewhat uncertain since only one line has been used in the analysis. The helium enrichment may indicate the evolved nature of the central star. 
Table 5a. Derived chemical composition of IRAS 13266-5551). The abundances are for $\log \epsilon(\mathrm{H})=12.0$. Solar abundances $\log \epsilon(\mathrm{X})_{\odot}$ are from Grevesse \& Sauval (1998) and Allende Prieto et al. (2001, 2002). $n$ refers to the number of lines of each species used for the abundance determination. $\sigma$ is the standard deviation. For comparison we have listed the average abundances of main sequence B-stars from the Ori OB1 association (Kilian 1992).

\begin{tabular}{|c|c|c|c|c|c|c|c|}
\hline \multirow[b]{2}{*}{$\mathrm{X}$} & \multicolumn{5}{|c|}{$\begin{array}{c}\text { IRAS } 13266-5551 \\
\left(T_{\mathrm{eff}}=23000 \mathrm{~K}, \log g=3.0, \xi_{\mathrm{t}}=10 \mathrm{~km} \mathrm{~s}^{-1}\right)\end{array}$} & \multirow[b]{2}{*}{$\log \epsilon(\mathrm{X})_{\odot}$} & \multirow{2}{*}{$\begin{array}{c}\text { Main sequence } \\
\text { B-stars, Ori OB1 } \\
\log \epsilon(\mathrm{X})\end{array}$} \\
\hline & $n$ & $\log \epsilon(X)$ & $\sigma$ & {$[\mathrm{X} / \mathrm{H}]$} & {$[\mathrm{X} / \mathrm{Fe}]$} & & \\
\hline $\mathrm{He} \mathrm{I}$ & 1 & $11.26^{\dagger}$ & & +0.33 & +0.50 & 10.93 & 11.04 \\
\hline C II & 4 & 8.67 & 0.27 & +0.28 & +0.45 & 8.39 & 8.23 \\
\hline N II & 6 & 8.23 & 0.29 & +0.31 & +0.48 & 7.92 & 7.72 \\
\hline O II & 4 & 8.78 & 0.28 & +0.09 & +0.26 & 8.69 & 8.60 \\
\hline $\mathrm{Ne} \mathrm{I}$ & 4 & $9.16^{*}$ & 0.32 & +1.08 & +1.25 & 8.08 & \\
\hline S II & 4 & $7.96^{\dagger}$ & & +0.63 & +0.80 & 7.33 & \\
\hline $\mathrm{Fe}$ III & 4 & 7.33 & 0.32 & -0.17 & & 7.50 & \\
\hline
\end{tabular}

The abundances were derived using Kurucz's WIDTH9 program (modified for Unix machines by John Lester at the University of Toronto, Canada).

$\dagger$ These values were derived from spectrum synthesis analysis using the SYNSPEC code.

* Ne abundances derived using Kurucz's WIDTH9 program appear to be in error. The observed Ne I lines are much stronger than the corresponding lines synthesised with the SYNSPEC code using the derived atmospheric parameters of the star and the Ne abundance in Table 5a. NLTE effects may be significant for these lines (see Sect. 3.8.5).

\subsubsection{II lines}

We observed 12 C II absorption lines in IRAS 13266-5551 and 6 C II absorption lines in IRAS 17311-4924. Some of these lines are weak and are blended with other lines. The ISO spectrum of IRAS 17311-4924 (Gauba \& Parthasarathy 2004) indicated carbon-rich circumstellar dust. The presence of carbon lines and their strengths compared to that of standard stars in the UV (Gauba \& Parthasarathy 2003) and optical spectra of this star indicate normal or slight overabundance of carbon.

\subsection{3. $N$ II lines}

Since strong lines $\left(W_{\lambda} \geq 100 \mathrm{~m} \AA\right)$ are usually affected by microturbulence, the use of these lines in determining the atmospheric parameters of the star may contribute to systematic errors. Hence N II lines with $W_{\lambda}>100 \mathrm{~m} \AA$ have been excluded from the abundance analysis of IRAS 13266-5551.

\subsubsection{O I triplet and O II lines}

The equivalent widths of the $\mathrm{O}$ I triplet in the spectra of the hot post-AGB stars, LSII $+34^{\circ} 26$ (B1.5 Ia, García-Lario et al. 1997; Arkhipova et al. 2001b) and IRAS 01005+7910 (Klochkova et al. 2002) are $0.95 \AA$ and $0.75 \AA$ respectively. The (total) equivalent widths of the O I triplet in IRAS 13266-5551 and IRAS $17311-4924$ are $0.741 \AA$ and $1.479 \AA$ respectively (Tables $2 \mathrm{a}$ and $3 \mathrm{a}$ ). The O I triplet at $\lambda 7773 \AA$ is known to be sensitive to NLTE effects. For the atmospheric parameters of IRAS 13266-5551, using LTE analysis, we attempted to synthesise the $\mathrm{O}$ I triplet with the SYNSPEC code. This required oxygen abundances $(\log \epsilon(\mathrm{O}))$ in excess of 10.5. In contrast, the derived oxygen abundance using LTE analysis for the O II lines in IRAS 13266-5551 is 8.78 (Table 5a). Such large discrepancies between the oxygen abundances derived from the O I triplet and the O II lines have also been observed in the hot post-AGB star IRAS $01005+7910$ (Klochkova et al. 2002).

The O II lines at $5161.349 \AA\left(W_{\lambda}=188.3 \mathrm{~m} \AA\right)$, $5208.14\left(W_{\lambda}=81.1 \mathrm{~m} \AA\right)$ and $6723.222 \AA\left(W_{\lambda}=168.5 \mathrm{~m} \AA\right)$ in IRAS 13266-5551 may also have significant NLTE effects. We could not obtain a good fit to these lines and they were excluded from the abundance analysis.

\subsubsection{Ne I lines}

The derived neon abundance for IRAS 13266-5551 is unusually high (Table 5a). However, we could still not obtain a good fit to the majority of Ne I lines in the spectrum of this star. Auer \& Mihalas (1973) showed that for stars in the range B2 to B5 the neon abundance deduced from LTE analyses is systematically in error by about a factor of five. They computed equivalent widths of Ne I lines ( $\lambda \lambda 5852.5 \AA$ to $6598.9 \AA$ ) for $15000 \mathrm{~K} \leq T_{\text {eff }} \leq 22500 \mathrm{~K}, \log g=3.0$ and 4.0 and solar neon abundance. For $T_{\text {eff }}=22500 \mathrm{~K}, \log g=3.0$, they found that the LTE equivalent widths were almost a factor of three smaller than the NLTE equivalent widths of these lines.

\subsubsection{Al III and Si III lines}

Only two lines of Al III and one of Si III were identified in each of the two stars. These lines are very strong (ref. Tables $2 \mathrm{a}$ and 3a) in both the stars. For instance, in IRAS 13266-5551, Si III, $5741.264 \AA$ has $W_{\lambda}=392.9 \mathrm{~m} \AA$. Using WIDTH9 and spectrum synthesis we derived $\log \epsilon(\mathrm{Al})=7.91 \pm 0.34$ and $\log \epsilon(\mathrm{Si})=9.23$ for IRAS 13266-5551. However, these abundances may be overestimates. 
Table 5b. Uncertainties in the abundances, $\Delta \log \epsilon(X)$ due to uncertainties in the the model atmospheric parameters.

\begin{tabular}{ccccc}
\hline \hline Element & $\begin{array}{c}\Delta T_{\text {eff }} \\
+1000 \mathrm{~K}\end{array}$ & $\begin{array}{c}\Delta \log g \\
+0.5\end{array}$ & $\begin{array}{c}\Delta \xi_{\mathrm{t}} \\
+1 \mathrm{~km} \mathrm{~s}^{-1}\end{array}$ & $\sigma_{\mathrm{m}}$ \\
\hline $\mathrm{C}$ & +0.12 & -0.18 & -0.02 & 0.22 \\
$\mathrm{~N}$ & +0.12 & -0.04 & -0.02 & 0.13 \\
$\mathrm{O}$ & -0.03 & +0.21 & -0.02 & 0.21 \\
$\mathrm{Ne}$ & +0.16 & -0.20 & -0.02 & 0.26 \\
$\mathrm{Fe}$ & +0.14 & +0.05 & -0.02 & 0.15 \\
\hline
\end{tabular}

\subsubsection{S II lines}

S II absorption lines were identified in IRAS 13266-5551. Since all except one S II line (5475.025 ̊) were identified as blends, we used the spectrum synthesis code SYNSPEC to estimate the sulphur abundance in this star (Table 5a). This value may therefore be treated as an upper limit.

\subsubsection{Iron lines}

From the Fe III absorption lines we estimated $[\mathrm{Fe} / \mathrm{H}]=$ -0.17 in the case of IRAS 13266-5551. The iron lines in IRAS 17311-4924 only appear in emission or as P-Cygni profiles.

\subsubsection{Estimated errors}

The standard deviations $(\sigma)$ measure the scatter in the abundances due to individual lines of a species. Table 5a gives the value of $\sigma$ for each species as estimated using WIDTH9. The true error, i.e. the standard deviation of the mean $\sigma / \sqrt{(} n)$, would be smaller for species with a greater number of lines (n).

Kurucz's solar metallicity models in the range $18000 \mathrm{~K} \leq$ $\mathrm{T}_{\text {eff }} \leq 25000 \mathrm{~K}$ are available only in steps of $\Delta T_{\text {eff }}= \pm 1000 \mathrm{~K}$ and $\Delta \log g= \pm 0.5$. Hence, the temperature $\left(T_{\text {eff }}\right)$ and gravity $(\log g)$ of IRAS $13266-5551$ are estimated to an accuracy of $\pm 1000 \mathrm{~K}$ and \pm 0.5 respectively. Table $5 \mathrm{~b}$ gives the uncertainties in the abundances due to uncertainties in the model atmospheric parameters taking $\Delta T_{\text {eff }}=+1000 \mathrm{~K}, \Delta \log g=+0.5$ and $\Delta \xi_{\mathrm{t}}=+1 \mathrm{~km} \mathrm{~s}^{-1}$. The quadratic sum of the uncertainities due to the model parameters is given by $\sigma_{\mathrm{m}}$ (Table $5 \mathrm{~b}$ ).

\section{Discussion and conclusions}

From LTE analysis of the high resolution optical spectrum of IRAS 13266-5551 we find the atmospheric parameters to be $T_{\text {eff }}=23000 \mathrm{~K} \pm 1000 \mathrm{~K}, \log g=3.0 \pm 0.5$ and $\xi_{\mathrm{t}}=10 \mathrm{~km} \mathrm{~s}^{-1}$. The lines of Ne I, a few O II lines and the O I triplet indicate that NLTE effects may be significant in these stars. Also, as they are hot stars it is important to obtain observations shortward of $4900 \AA$. The absorption lines in the blue may significantly improve our estimates of the stellar parameters and elemental abundances. Mooney et al. (2002), Klochkova et al. (2002) and Ryans et al. (2003) among others have obtained observations from $\sim 3700 \AA$ onwards. Recently, from NLTE analysis of the absorption lines in the blue region, Ryans et al. (2003) estimated the atmospheric parameters of two hot post-AGB stars, IRAS $18062+2410$ (SAO85766) and IRAS 19590-1249 (LSIV-12 ${ }^{\circ} 111$ ). A similar analysis is required for IRAS 13266-5551 and IRAS 17311-4924. Hence, we would like to emphasise that our LTE analysis based on lines longward of $4900 \AA$ is only a first approximation.

We estimated heliocentric radial velocities $\left(V_{\mathrm{r}}\right)$ of $+65.31 \pm$ $0.34 \mathrm{~km} \mathrm{~s}^{-1}$ and $+27.55 \pm 0.74 \mathrm{~km} \mathrm{~s}^{-1}$ for IRAS 13266-5551 and IRAS 17311-4924 respectively.

Preliminary estimates of the $\mathrm{CNO}$ abundances in IRAS 13266-5551 indicate that these elements are overabundant with $[\mathrm{C} / \mathrm{Fe}]=+0.45$, $[\mathrm{N} / \mathrm{Fe}]=+0.48$ and $[\mathrm{O} / \mathrm{Fe}]=$ +0.26 suggesting that the products of helium burning have been brought to the surface as a result of third dredge-up on the AGB. A comparison with average $\mathrm{CNO}$ abundances for main-sequence B-stars from the Ori OB1 association (Table 5a; Kilian 1992) also indicates that IRAS 13266-5551 is an evolved star and has gone through the dredge-up episodes during its evolution. We could not estimate the atmospheric parameters and chemical abundances for IRAS 17311-4924.

McCausland et al. (1992) and Conlon et al. (1993b) derived the chemical composition of several high-Galactic-latitude hot post-AGB stars. In addition to being metal-poor, these stars showed severe carbon deficiencies. Similar carbon depletions were also reported in other hot post-AGB stars at high Galactic latitudes e.g. LSII $+34^{\circ} 26$ (García-Lario et al. 1997), PG1323-086 and PG1704+222 (Moehler \& Heber 1998), and SAO85766 (Parthasarathy et al. 2000b). In contrast, the hot post-AGB star, IRAS $01005+7910$ (Klochkova et al. 2002) was found to be carbon-rich. For IRAS 13266-5551 we estimated $\mathrm{C} / \mathrm{O} \sim 0.78$.

Finally, from our optical spectra we conclude that IRAS 13266-5551 and IRAS 17311-4924 are most likely in the post-AGB phase of evolution. These stars are unlikely to be luminous blue variables (LBVs). Their spectra are very similar to the hot post-AGB stars IRAS 18062+2410 (SAO85766; Parthasarathy et al. 2000b) and IRAS 01005+7910 (Klochkova et al. 2002). LBVs are usually found in the Galactic plane and are often associated with star forming regions. IRAS 13266-5551 and IRAS 17311-4924 on the other hand, are at high Galactic latitudes and are not associated with any star forming region. Further, whereas LBVs are characterised by large-amplitude light variations, these stars may show small-amplitude irregular light variations similar to those found in the high-Galactic-latitude rapidly evolving hot postAGB star, SAO85766 (Arkhipova 1999, 2000). Photometric monitoring of these stars is required. 
Acknowledgements. We would like to thank Dr. John Lester at the University of Toronto, Canada for having kindly provided the Unix version of the WIDTH9 program. We would also like to thank the referee, Dr. V. G. Klochkova for helpful comments.

\section{References}

Allende Prieto, C., Lambert, D. L., \& Asplund, M. 2001, ApJ, 556, L63

Allende Prieto, C., Lambert, D. L., \& Asplund, M. 2002, ApJ, 573, L137

Arkhipova, V. P., Ikonnikova, N. P., Noskova, R. I., et al. 1999, Astro. Lett., 25, 25

Arkhipova, V. P., Ikonnikova, N. P., Noskova, R. I., \& Sokol, G. V. 2000, Astro. Lett., 26, 609

Arkhipova, V. P., Klochkova, V. G., \& Sokol, G. V. 2001a, Astro. Lett., 27,99

Arkhipova, V. P., Ikonnikova, N. P., Noskova, R. I., et al. 2001b, Astro. Lett., 27, 719

Auer, L. H., \& Mihalas, D. 1973, ApJ, 184, 151

Conlon, E. S., Dufton, P. L., McCausland, R. J. H., et al. 1993a, ApJ, 408, 593

Conlon, E. S., McCausland, R. J. H., Dufton, P. L., \& Keenan, F. P. 1993b, in Luminous High-Latitude Stars, ed. D. D. Sasselov, ASP Conf. Ser., 45, 146

Conti, P. S., \& Frost, S. A. 1977, ApJ, 212, 728

Ebbets, D. 1982, ApJS, 48, 399

García-Lario, P. Parthasarathy, M., de Martino, D., et al. 1997, A\&A, 326,1103

Gauba, G., \& Parthasarathy, M. 2003, A\&A, 407, 1007

Gauba, G., \& Parthasarathy, M. 2004, A\&A, 417, 201

Grevesse, N., \& Sauval, A. J. 1998, Space Sci. Rev., 85, 161

Herbig, G. H. 1993, ApJ, 407, 142

Hony, S., Waters, L. B. F. M., \& Tielens, A. G. G. M. 2002, A\&A, 390, 533

Hrivnak, B. J., Volk, K., \& Kwok, S. 2000, ApJ, 535, 275

Hubeny, I., Stefl, S., \& Harmanec, P. 1985, Bull. Astron. Inst. Czechosl., 36, 214

Kilian, J. 1992, A\&A, 262, 171

Klein, R. I., \& Castor, J. I. 1978, ApJ, 220, 902

Klochkova, V. G., Yushkin, M. V., Miroshnichenko, A. S., et al. 2002, A\&A, 392, 143

Kozok, J. R. 1985, A\&AS, 61, 387

Kurucz, R. L. 1994, Solar Abundance Model Atmospheres, Kurucz CDROM No. 19, Smithsonian Astrophysical Observatory
Lang, K. R. 1992, Astrophysical Data: Planets and Stars (Springer-Verlag)

Leitherer, C. 1988, ApJ, 326, 356

Loup, C., Forveille, T., Nyman, L. A., \& Omont, A. 1990, A\&A, 227, L29

McCausland, R. J. H., Conlon, E. S., Dufton, P. L., et al. 1992, ApJ, 394, 298

Miroshnichenko, A. S., Fremat, I., Houziaux, L., et al. 1998, A\&AS, 131,469

Moehler, S., \& Heber, U. 1998, A\&A, 335, 985

Mooney, C. J., Rolleston, W. R. J., Keenan, F. P., et al. 2002, MNRAS, 337,851

Moore, C. E. 1945, A multiplet table of astrophysical interest

Nyman, L. A., Booth, R. S., Carlstrom, U., et al. 1992, A\&AS, 93, 121

Osterbrock, D. E., \& Martel, A. 1992, PASP, 104, 76

Osterbrock, D. E., Fulbright, J. P., Martel, A., et al. 1996, PASP, 108, 277

Parthasarathy, M. 1993a, in Luminous High-Latitude Stars, ed. D. D. Sasselov, ASP Conf. Ser., 45, 173

Parthasarathy, M. 1993b, ApJ, 414, L109

Parthasarathy, M., \& Pottasch, S. R. 1986, A\&A, 154, L16

Parthasarathy, M., \& Pottasch, S. R. 1989, A\&A, 225, 521

Parthasarathy, M., García-Lario, P., \& Pottasch, S. R. 1992, A\&A, 264, 159

Parthasarathy, M., García-Lario, P., Pottasch, S. R., et al. 1993, A\&A, 267, L19

Parthasarathy, M., García-Lario P., de Martino, D., et al 1995, A\&A, 300, L25

Parthasarathy, M., Vijapurkar, J., \& Drilling, J. S. 2000a, A\&AS, 145, 269

Parthasarathy, M., García-Lario, P., Sivarani, T., et al. 2000b, A\&A, 357,241

Parthasarathy, M., Gauba, G., Fujii, T., \& Nakada, Y. 2001, in Post-AGB Objects as a Phase of Stellar Evolution, ed. R. Szczerba, \& S. K. Górny (Boston/Dordrecht/London: Kluwer Academic Publishers), Astrophysics and Space Science Library, 265,29

Reed, B. C. 1998, ApJS, 115, 271

Ryans, R. S. I., Dufton, P. L., Mooney, C. J., et al. 2003, A\&A, 401, 1119

Schlegel, D. J., Finkbeiner, D. P., \& Davis, M. 1998, ApJ, 500, 525

Volk, K., Xiong, G. Z., \& Kwok, S. 2000, ApJ, 530, 408

Volk, K., Kwok, S., \& Hrivnak, B. J. 2002, ApJ, 567, 412

Weinberger, R. 1989, A\&AS, 78, 301 
G. Sarkar et al.: Hot post-AGB stars: IRAS 13266-5551 and IRAS 17311-4924, Online Material p 1

\section{Online Material}


Table 2a. Absorption lines in IRAS 13266-5551 (CPD-55 5588).

\begin{tabular}{|c|c|c|c|c|c|c|c|}
\hline $\begin{array}{l}\lambda_{\text {obs. }} \\
(\AA)\end{array}$ & $\begin{array}{l}\lambda_{\text {lab. }} \\
(\AA)\end{array}$ & Ident. & $\begin{array}{l}W_{\lambda} \\
(\AA)\end{array}$ & $\overline{\log (g f)}$ & $\begin{array}{c}\chi \\
(\mathrm{eV})\end{array}$ & $\begin{array}{l}\Delta \lambda \\
(\AA)\end{array}$ & $\begin{array}{c}V_{\mathrm{r}} \\
\mathrm{km} \mathrm{s}^{-1}\end{array}$ \\
\hline 4942.394 & 4941.105 & O II (33) & 0.0498 & 0.080 & $26.55-29.06$ & 1.289 & +63.03 \\
\hline 4944.33 & 4943.003 & O II (33) & 0.0901 & 0.370 & $26.56-29.07$ & 1.327 & +65.31 \\
\hline 4957.005 & 4955.738 & O II (33) & 0.0437 & -0.420 & $26.56-29.06$ & 1.267 & +61.47 \\
\hline 4995.447 & 4994.360 & N II $(24,64)$ & 0.028 & -0.080 & $20.94-23.42$ & & \\
\hline 5002.728 & 5001.474 & N II (19) & 0.222 & 0.450 & $20.65-23.13$ & & blend \\
\hline 5003.995 & 5002.703 & N II (4) & 0.0665 & -1.020 & $18.46-20.94$ & & blend \\
\hline 5006.535 & 5005.150 & N II $(19,6)$ & 0.1499 & 0.610 & $20.66-23.14$ & & \\
\hline 5008.656 & 5007.328 & N II (24) & 0.0494 & 0.160 & $20.94-23.41$ & 1.328 & +64.33 \\
\hline 5011.923 & 5010.621 & N II (4) & 0.1593 & -0.520 & $18.46-20.94$ & 1.302 & +62.72 \\
\hline 5046.411 & 5045.099 & N II (4) & 0.1784 & -0.330 & $18.48-20.94$ & 1.312 & +62.79 \\
\hline 5049.117 & 5047.738 & He I (47) & 0.1329 & -1.600 & $21.22-23.67$ & 1.379 & +66.73 \\
\hline \multirow[t]{2}{*}{5075.435} & 5073.592 & N II (10) & & -1.280 & $18.49-20.94$ & & \\
\hline & +5073.903 & Fe III (5) & & -2.557 & $8.65-11.09$ & & \\
\hline 5088.355 & 5086.701 & Fe III (5) & 0.0372 & -2.590 & $8.65-11.09$ & 1.654 & +82.32 \\
\hline 5129.21 & 5127.387 & Fe III (5) & 0.0390 & -2.218 & $8.65-11.07$ & 1.823 & +91.43 \\
\hline \multirow{2}{*}{5134.133} & 5132.947 & C II (16) & 0.102 & -0.240 & $20.70-23.12$ & & \\
\hline & +5133.281 & C II (16) & & -0.200 & $20.70-23.12$ & & \\
\hline 5144.795 & 5143.494 & C II (16) & 0.045 & -0.240 & $20.70-23.11$ & 1.301 & +60.65 \\
\hline 5146.582 & 5145.165 & C II (16) & 0.0896 & 0.160 & $20.71-23.12$ & 1.417 & +67.39 \\
\hline 5152.49 & 5151.085 & C II (16) & 0.0781 & -0.200 & $20.71-23.12$ & 1.405 & +66.60 \\
\hline 5157.759 & 5156.111 & Fe III (5) & 0.0398 & -2.018 & $8.64-11.04$ & 1.648 & +80.66 \\
\hline 5161.349 & 5160.026 & O II (32) & 0.1883 & -0.660 & $26.56-28.96$ & 1.323 & +61.69 \\
\hline 5208.14 & 5206.715 & O II (32) & 0.0811 & -0.860 & $26.56-28.94$ & 1.425 & +66.87 \\
\hline 5220.938 & & UN & 0.133 & & & & \\
\hline \multirow[t]{2}{*}{5455.271} & 5453.790 & S II (6) & 0.1352 & 0.560 & $13.67-15.94$ & & \\
\hline & +5454.215 & N II (29) & & -0.740 & $21.15-23.42$ & & \\
\hline 5475.025 & 5473.602 & S II (6) & 0.0982 & -0.120 & $13.58-15.85$ & 1.423 & +62.76 \\
\hline 5497.073 & 5495.655 & N II (29) & 0.0767 & -0.170 & $21.16-23.42$ & 1.418 & +62.18 \\
\hline \multirow[t]{2}{*}{5641.593} & 5639.980 & S II (14) & 0.0480 & 0.330 & $14.07-16.26$ & & \\
\hline & +5640.549 & C II (15) & & -0.750 & $20.70-22.90$ & & \\
\hline \multirow[t]{2}{*}{5648.699} & 5646.979 & S II (14) & 0.0426 & 0.110 & $14.00-16.20$ & & \\
\hline & +5648.070 & C II (15) & & -0.450 & $20.70-22.90$ & & \\
\hline 5668.134 & 5666.629 & N II (3) & 0.2938 & 0.010 & $18.47-20.65$ & 1.505 & +64.45 \\
\hline 5677.53 & 5676.017 & N II (3) & 0.2524 & -0.340 & $18.46-20.65$ & 1.513 & +64.74 \\
\hline 5681.04 & 5679.558 & N II (3) & 0.3913 & 0.280 & $18.48-20.67$ & 1.482 & +63.05 \\
\hline 5687.75 & 5686.213 & N II (3) & 0.1746 & -0.470 & $18.47-20.65$ & 1.537 & +65.86 \\
\hline 5698.092 & 5696.603 & Al III (2) & 0.3473 & 0.230 & $15.64-17.82$ & 1.489 & +63.19 \\
\hline 5712.274 & 5710.766 & N II (3) & 0.1726 & -0.470 & $18.48-20.65$ & 1.508 & +63.99 \\
\hline 5724.268 & 5722.730 & Al III (2) & 0.2174 & -0.070 & $15.64-17.81$ & 1.538 & +65.39 \\
\hline 5741.264 & 5739.734 & Si III (4) & 0.3929 & -0.160 & $19.72-21.88$ & 1.53 & +64.74 \\
\hline 5780.631 & 5780.410 & DIB & 0.1696 & & & 0.221 & -3.76 \\
\hline 5797.34 & 5797.030 & DIB & 0.0998 & & & 0.310 & +0.81 \\
\hline 5835.532 & 5833.938 & Fe III (114) & 0.1198 & 0.616 & $18.51-20.63$ & 1.594 & +66.74 \\
\hline 5933.462 & 5931.782 & N II (28) & 0.0428 & 0.050 & $21.15-23.24$ & 1.68 & +69.74 \\
\hline 5943.301 & 5941.654 & N II (28) & 0.0756 & 0.320 & $21.16-23.25$ & 1.647 & +67.93 \\
\hline 6144.637 & 6143.063 & $\mathrm{Ne} I(1)$ & 0.0606 & -0.350 & $16.62-18.64$ & 1.574 & +61.64 \\
\hline 6196.062 & 6195.990 & DIB & 0.0214 & & & 0.072 & -11.74 \\
\hline 6203.327 & 6203.060 & DIB & 0.0221 & & & 0.267 & -2.32 \\
\hline 6381.399 & 6379.617 & N II (2) & 0.0587 & -0.920 & $18.47-20.41$ & 1.782 & +68.57 \\
\hline
\end{tabular}


G. Sarkar et al.: Hot post-AGB stars: IRAS 13266-5551 and IRAS 17311-4924, Online Material p 3

Table 2a. continued.

\begin{tabular}{cccccccc}
\hline \hline $\begin{array}{c}\lambda_{\text {obs. }} \\
(\AA)\end{array}$ & $\begin{array}{c}\lambda_{\text {lab. }} \\
(\AA)\end{array}$ & Ident. & $\begin{array}{c}W_{\lambda} \\
(\AA)\end{array}$ & $\log (g f)$ & $\begin{array}{c}\chi \\
(\mathrm{eV})\end{array}$ & $\begin{array}{c}\Delta \lambda \\
(\AA)\end{array}$ & $\begin{array}{c}V_{\mathrm{r}} \\
\mathrm{km} \mathrm{s}^{-1}\end{array}$ \\
\hline 6403.899 & 6402.246 & Ne I (1) & 0.1314 & 0.360 & $16.62-18.55$ & 1.653 & +62.23 \\
6483.787 & 6482.049 & N II (8) & 0.2212 & -0.160 & $18.50-20.41$ & 1.738 & +65.21 \\
6508.432 & 6506.528 & Ne I (3) & 0.0275 & 0.030 & $16.67-18.58$ & 1.904 & +72.56 \\
6579.07 & $6578.052^{\dagger}$ & C II (2) & 0.0855 & 0.120 & $14.45-16.33$ & 1.018 & +46.43 \\
6613.856 & 6613.630 & DIB & 0.0832 & & & 0.226 & -4.98 \\
6642.826 & 6640.994 & O II (4) & 0.0825 & -0.890 & $23.42-25.29$ & 1.832 & +67.53 \\
6723.222 & 6721.358 & O II (4) & 0.1685 & -0.590 & $23.44-25.29$ & 1.864 & +67.97 \\
6781.99 & 6779.942 & C II (14) & 0.0355 & 0.040 & $20.70-22.53$ & & \\
& +6780.595 & C II (14) & & -0.360 & $20.70-22.53$ & & \\
6785.831 & 6783.907 & C II (14) & 0.0458 & 0.320 & $20.71-22.54$ & 1.924 & +69.85 \\
& 6787.210 & C II (14) & & -0.360 & $20.70-22.53$ & & Weak \\
& 6791.465 & C II (14) & & -0.250 & $20.70-22.53$ & & Weak \\
& 6800.687 & C II (14) & & -0.330 & $20.70-22.54$ & & Weak \\
7034.428 & 7032.413 & Ne I (1) & 0.0485 & -0.250 & $16.62-18.38$ & 2.015 & +70.73 \\
7773.521 & 7771.944 & O I (1) & 0.273 & 0.320 & $9.14-10.74$ & & Blend \\
7776.411 & 7774.166 & O I (1) & 0.396 & 0.170 & $9.14-10.74$ & & Blend \\
7777.68 & 7775.388 & O I (1) & 0.072 & -0.050 & $9.14-10.74$ & & Weak \\
\hline
\end{tabular}

† The radial velocity of the C II(2) $6578.052 \AA$ absorption line indicates that it may have a P-Cygni profile with a weak emission component, similar to C II(2) $6582.882 \AA$ (see Table 2c). 
Table 2b. Emission lines in IRAS 13266-5551 (CPD-55 5588).

\begin{tabular}{|c|c|c|c|c|c|c|c|}
\hline $\begin{array}{l}\lambda_{\text {obs. }} \\
(\AA)\end{array}$ & $\begin{array}{l}\lambda_{\text {lab. }} \\
(\AA)\end{array}$ & Ident. & $\begin{array}{l}W_{\lambda} \\
(\AA)\end{array}$ & $\log (g f)$ & $\begin{array}{c}\chi \\
(\mathrm{eV})\end{array}$ & $\begin{array}{l}\Delta \lambda \\
(\AA)\end{array}$ & $\begin{array}{c}V_{\mathrm{r}} \\
\mathrm{km} \mathrm{s}^{-1}\end{array}$ \\
\hline & $5015.678^{\dagger}$ & $\mathrm{He} \mathrm{I} \mathrm{(4)}$ & & -0.820 & $20.62-23.09$ & & \\
\hline 5042.128 & 5041.024 & Si II (5) & 0.0913 & 0.290 & $10.07-12.52$ & 1.104 & +50.47 \\
\hline 5057.172 & 5055.984 & Si II (5) & 0.1503 & 0.590 & $10.07-12.52$ & 1.188 & +55.26 \\
\hline 5199.059 & & UN & 0.0281 & & & & \\
\hline 5201.533 & & UN & 0.0263 & & & & \\
\hline 5467.921 & 5466.55 & S II (11) & 0.0346 & & $13.62-15.88$ & 1.371 & +60.01 \\
\hline 5516.046 & & UN & 0.0924 & & & & \\
\hline 5921.673 & 5920.124 & Fe III (115) & 0.0420 & -0.034 & $18.78-20.88$ & 1.549 & +63.26 \\
\hline 5955.113 & 5953.613 & Fe III (115) & 0.0371 & 0.186 & $18.79-20.87$ & 1.5 & +60.35 \\
\hline 5958.987 & 5957.559 & Si II (4) & 0.1042 & -0.300 & $10.07-12.15$ & 1.428 & +56.68 \\
\hline \multirow[t]{2}{*}{5980.413} & 5978.90 & Fe III (117) & 0.3632 & & $18.73-20.80$ & & \\
\hline & +5978.930 & Si II (4) & & 0.000 & $10.07-12.15$ & & \\
\hline 6000.962 & 5999.30 & Fe III (117) & 0.0328 & & $18.73-20.79$ & 1.662 & +67.88 \\
\hline 6033.913 & 6032.30 & Fe III (117) & 0.0666 & & $18.73-20.78$ & 1.613 & +64.99 \\
\hline 6096.85 & 6095.37 & C II (24) & 0.0226 & & $22.47-24.50$ & 1.48 & +57.61 \\
\hline 6100.046 & 6098.62 & C II (24) & 0.0280 & & $22.47-24.50$ & 1.426 & +54.92 \\
\hline 6227.758 & 6226.130 & Al II (10) & 0.0100 & 0.050 & $13.07-15.06$ & 1.628 & +63.21 \\
\hline 6233.21 & 6231.718 & Al II (10) & 0.0421 & 0.400 & $13.07-15.06$ & 1.492 & +56.6 \\
\hline 6241.053 & & UN & 0.0398 & & & & \\
\hline 6244.939 & 6243.355 & Al II (10) & 0.0281 & 0.670 & $13.08-15.06$ & 1.584 & +60.88 \\
\hline 6300.392 & & [O I ] (1F) (atmos.) & 0.0728 & & & & \\
\hline 6348.553 & 6347.091 & Si II (2) & 0.2767 & 0.300 & $8.12-10.07$ & 1.462 & +53.87 \\
\hline 6363.881 & & [O I] (1F) (atmos.) & 0.0222 & & & & \\
\hline 6372.811 & 6371.359 & Si II (2) & 0.1369 & 0.000 & $8.12-10.07$ & 1.452 & +53.14 \\
\hline 6463.481 & & Atmos. & 0.0399 & & & & \\
\hline 6547.435 & 6545.80 & Mg II (23) & 0.0303 & & $11.58-13.47$ & 1.635 & +59.70 \\
\hline \multirow[t]{2}{*}{6549.576} & 6548.1 & {$[\mathrm{~N} \mathrm{II}](1 \mathrm{~F})$} & 0.0209 & & & 1.476 & +52.39 \\
\hline & $6583.6^{*}$ & {$[\mathrm{~N} \mathrm{II}](1 \mathrm{~F})$} & & & & & \\
\hline 6732.171 & & UN & 0.0259 & & & & \\
\hline 6853.447 & & UN & 0.0463 & & & & \\
\hline 7043.729 & 7042.048 & Al II (3) & 0.0818 & 0.350 & $11.32-13.08$ & 1.681 & +56.38 \\
\hline 7058.222 & 7056.612 & Al II (3) & 0.0619 & 0.130 & $11.32-13.07$ & 1.61 & +53.22 \\
\hline 7063.996 & & UN & 0.016 & & & & \\
\hline 7065.332 & 7063.642 & Al II (3) & 0.0471 & -0.350 & $11.32-13.07$ & 1.68 & +56.12 \\
\hline \multirow[t]{2}{*}{7067.219} & 7065.188 & He I (10) & 0.5544 & & $20.87-22.62$ & & \\
\hline & +7065.719 & He I (10) & & & $20.87-22.62$ & & \\
\hline 7233.203 & 7231.332 & C II (3) & 0.316 & 0.070 & $16.33-18.05$ & 1.871 & +62.39 \\
\hline \multirow[t]{2}{*}{7238.37} & 7236.421 & C II (3) & 0.488 & 0.330 & $16.33-18.05$ & 1.949 & +65.57 \\
\hline & $7281.349^{\dagger}$ & $\mathrm{He} \mathrm{I} \mathrm{(45)}$ & & -0.840 & $21.22-22.92$ & & \\
\hline
\end{tabular}

† The He I(4) $5015.675 \AA$ and He I(45) $7281.349 \AA$ emission lines are superposed on the corresponding absorption profiles of these lines. The asymmetric nature of these emission lines suggests that they may have P-Cygni profiles.

* [N II] (1F) $6583.6 \AA$ is blended with the emission component of the C II (2) $6582.882 \AA$ P-Cygni profile. 
G. Sarkar et al.: Hot post-AGB stars: IRAS 13266-5551 and IRAS 17311-4924, Online Material p 5

Table 2b. continued.

\begin{tabular}{|c|c|c|c|c|c|c|c|}
\hline $\begin{array}{l}\lambda_{\text {obs. }} \\
(\AA)\end{array}$ & $\begin{array}{l}\lambda_{\text {lab. }} \\
(\AA)\end{array}$ & Ident. & $\begin{array}{l}W_{\lambda} \\
(\AA)\end{array}$ & $\log (g f)$ & $\begin{array}{c}\chi \\
(\mathrm{eV})\end{array}$ & $\begin{array}{l}\Delta \lambda \\
(\AA)\end{array}$ & $\begin{array}{c}V_{\mathrm{r}} \\
\mathrm{km} \mathrm{s}^{-1}\end{array}$ \\
\hline 7316.384 & & Atmos. & 0.0237 & & & & \\
\hline 7379.562 & & UN & 0.0736 & & & & \\
\hline 7413.63 & & Atmos. & 0.0258 & & & & \\
\hline 7464.238 & & UN & 0.0624 & & & & \\
\hline 7468.316 & & Atmos. & 0.0306 & & & & \\
\hline 7497.305 & & $\mathrm{UN}$ & 0.0161 & & & & \\
\hline 7504.157 & & UN & 0.0123 & & & & \\
\hline 7514.875 & & UN & 0.0569 & & & & \\
\hline 7564.141 & & UN & 0.0178 & & & & \\
\hline 7712.676 & & Atmos. & 0.0276 & & & & \\
\hline 7717.028 & & Atmos. & 0.0126 & & & & \\
\hline 7750.781 & & Atmos. & 0.0322 & & & & \\
\hline 7794.201 & & Atmos. & 0.0223 & & & & \\
\hline 7821.623 & & Atmos. & 0.0517 & & & & \\
\hline 7851.066 & & UN & 0.1470 & & & & \\
\hline 7853.381 & & Atmos. & 0.0263 & & & & \\
\hline 7854.82 & & UN & 0.0248 & & & & \\
\hline 7878.902 & 7877.054 & Mg II (8) & 0.2393 & 0.390 & $9.99-11.57$ & 1.848 & +55.15 \\
\hline 7898.179 & 7896.367 & Mg II (8) & 0.3963 & 0.650 & $10.00-11.57$ & 1.812 & +53.61 \\
\hline 7913.766 & & Atmos. & 0.0636 & & & & \\
\hline 7921.164 & & Atmos. & 0.0420 & & & & \\
\hline 7964.76 & & Atmos. & 0.0554 & & & & \\
\hline 7993.434 & & Atmos. & 0.0468 & & & & \\
\hline 8001.991 & 8000.12 & {$[\mathrm{Cr} \mathrm{II}](1 \mathrm{~F})$} & 0.0441 & & & 1.871 & +54.93 \\
\hline 8025.808 & & Atmos. & 0.0350 & & & & \\
\hline 8062.346 & & Atmos. & 0.0199 & & & & \\
\hline 8215.857 & & UN & 0.0891 & & & & \\
\hline 8236.683 & & UN & 0.2139 & & & & \\
\hline
\end{tabular}


Table 3a. Absorption lines in IRAS 17311-4924 (Hen3-1428).

\begin{tabular}{|c|c|c|c|c|c|c|c|}
\hline $\begin{array}{l}\lambda_{\text {obs. }} \\
(\AA)\end{array}$ & $\begin{array}{l}\lambda_{\text {lab. }} \\
(\AA)\end{array}$ & Ident. & $\begin{array}{l}W_{\lambda} \\
(\AA)\end{array}$ & $\log (g f)$ & $\begin{array}{c}\chi \\
(\mathrm{eV})\end{array}$ & $\begin{array}{l}\Delta \lambda \\
(\AA)\end{array}$ & $\begin{array}{c}V_{\mathrm{r}} \\
\mathrm{km} \mathrm{s}^{-1}\end{array}$ \\
\hline \multirow[t]{2}{*}{5045.576} & 5044.8 & C II (35) & 0.0976 & & $24.27-26.71$ & & \\
\hline & +5045.099 & N II (4) & & -0.330 & $18.48-20.94$ & & \\
\hline 5047.665 & 5047.2 & C II (35) & 0.0686 & -1.00 & $24.27-26.71$ & 0.465 & +25.17 \\
\hline \multirow[t]{2}{*}{5133.486} & 5132.947 & C II (16) & 0.0643 & -0.240 & $20.70-23.12$ & & \\
\hline & +5133.281 & C II (16) & & -0.200 & $20.70-23.12$ & & \\
\hline 5144.009 & 5143.494 & C II (16) & 0.0436 & -0.240 & $20.70-23.12$ & 0.515 & +27.57 \\
\hline \multirow[t]{2}{*}{5145.53} & 5145.011 & $\mathrm{Ne} I$ (34) & 0.0913 & & $18.62-21.02$ & & \\
\hline & +5145.165 & C II (16) & & 0.160 & $20.71-23.12$ & & \\
\hline 5151.433 & 5151.085 & C II (16) & 0.0294 & -0.200 & $20.71-23.12$ & 0.348 & +17.8 \\
\hline $5160.659^{\dagger}$ & 5160.026 & O II (32) & 0.0584 & -0.660 & $26.55-28.96$ & & blend \\
\hline 5207.288 & 5206.715 & O II (32) & 0.0137 & -0.860 & $26.56-28.94$ & 0.573 & +30.54 \\
\hline 5496.109 & 5495.655 & N II (29) & 0.0215 & -0.170 & $21.16-23.42$ & 0.454 & +22.31 \\
\hline 5667.181 & 5666.629 & N II (3) & 0.1652 & 0.010 & $18.46-20.65$ & 0.552 & +26.75 \\
\hline 5676.614 & 5676.017 & N II (3) & 0.1386 & -0.340 & $18.46-20.65$ & 0.597 & +29.08 \\
\hline 5680.022 & 5679.558 & N II (3) & 0.2475 & 0.280 & $18.48-20.67$ & 0.464 & +22.04 \\
\hline 5686.792 & 5686.213 & N II (3) & 0.1017 & -0.470 & $18.47-20.65$ & 0.579 & +28.08 \\
\hline 5697.17 & 5696.603 & Al III (2) & 0.1941 & 0.230 & $15.64-17.82$ & 0.567 & +27.39 \\
\hline 5711.247 & 5710.766 & N II (3) & 0.0758 & -0.470 & $18.48-20.65$ & 0.481 & +22.80 \\
\hline 5723.368 & 5722.730 & Al III (2) & 0.1158 & -0.070 & $15.64-17.81$ & 0.638 & +30.97 \\
\hline 5740.416 & 5739.734 & Si III (4) & 0.1923 & -0.160 & $19.72-21.88$ & 0.682 & +33.18 \\
\hline 5780.096 & 5780.410 & DIB & 0.1099 & & & -0.314 & -18.77 \\
\hline 6143.323 & 6143.063 & $\mathrm{Ne} I$ (1) & 0.1289 & -0.350 & $16.62-18.64$ & 0.26 & +10.23 \\
\hline 6163.944 & 6163.594 & $\mathrm{Ne} I$ (5) & 0.0460 & -0.590 & $16.72-18.73$ & 0.35 & +14.56 \\
\hline 6266.823 & 6266.495 & $\mathrm{Ne} \mathrm{I} \mathrm{(5)}$ & 0.0351 & -0.530 & $16.72-18.69$ & 0.328 & +13.23 \\
\hline 6334.755 & 6334.428 & $\mathrm{Ne} I$ (1) & 0.0709 & -0.310 & $16.62-18.58$ & 0.327 & +13.02 \\
\hline 6380.267 & 6379.617 & N II (2) & 0.0216 & -0.920 & $18.47-20.41$ & 0.65 & +28.10 \\
\hline 6383.238 & 6382.991 & $\mathrm{Ne} I$ (3) & 0.0587 & -0.260 & $16.67-18.61$ & 0.247 & +9.14 \\
\hline 6402.463 & 6402.246 & $\mathrm{Ne} \mathrm{I}(1)$ & 0.2416 & 0.360 & $16.62-18.56$ & 0.217 & +7.7 \\
\hline 6482.705 & 6482.049 & N II (8) & 0.1270 & -0.160 & $18.50-20.41$ & 0.656 & +27.89 \\
\hline 6506.754 & 6506.528 & $\mathrm{Ne} I$ (3) & 0.0441 & 0.030 & $16.67-18.58$ & 0.226 & +7.95 \\
\hline 6641.812 & 6640.994 & O II (4) & 0.0738 & -0.890 & $23.42-25.29$ & 0.818 & +34.48 \\
\hline 6722.179 & 6721.358 & O II (4) & 0.1031 & -0.590 & $23.44-25.29$ & 0.821 & +34.17 \\
\hline 7032.735 & 7032.413 & $\mathrm{Ne} I$ (1) & 0.1089 & -0.250 & $16.62-18.38$ & 0.322 & +11.27 \\
\hline 7772.134 & 7771.944 & O I (1) & 0.743 & 0.320 & $9.14-10.74$ & & Blend \\
\hline 7774.474 & 7774.166 & O I (1) & 0.530 & 0.170 & $9.14-10.74$ & & Blend \\
\hline 7775.758 & 7775.388 & O I (1) & 0.206 & -0.050 & $9.14-10.74$ & & Blend \\
\hline
\end{tabular}

† The O II (32) $5160.026 \AA$ absorption line is blended with [Fe II] (19F) $5158.81 \AA$. 
G. Sarkar et al.: Hot post-AGB stars: IRAS 13266-5551 and IRAS 17311-4924, Online Material p 7

Table 3b. Emission lines in IRAS 17311-4924 (Hen3-1428).

\begin{tabular}{|c|c|c|c|c|c|c|c|}
\hline $\begin{array}{c}\lambda_{\text {obs. }} \\
(\AA)\end{array}$ & $\begin{array}{l}\lambda_{\text {lab. }} \\
(\AA)\end{array}$ & Ident. & $\begin{array}{l}W_{\lambda} \\
(\AA)\end{array}$ & $\log (g f)$ & $\begin{array}{c}\chi \\
(\mathrm{eV})\end{array}$ & $\begin{array}{l}\Delta \lambda \\
(\AA)\end{array}$ & $\begin{array}{c}V_{\mathrm{r}} \\
\mathrm{km} \mathrm{s}^{-1}\end{array}$ \\
\hline 5041.596 & 5041.024 & Si II (5) & 0.0952 & 0.290 & $10.07-12.53$ & 0.572 & +31.57 \\
\hline 5056.559 & 5055.984 & Si II (5) & 0.1625 & 0.590 & $10.07-12.53$ & 0.575 & +31.65 \\
\hline 5122.428 & 5121.69 & C II (12) & 0.0712 & & $20.06-23.47$ & 0.738 & +40.76 \\
\hline 5159.448 & 5158.81 & {$[\mathrm{Fe} I \mathrm{II}](19 \mathrm{~F})$} & 0.049 & & & 0.638 & +34.63 \\
\hline 5194.567 & 5193.909 & Fe III (5) & 0.0186 & -2.852 & $8.66-11.04$ & 0.658 & +35.54 \\
\hline 5198.512 & 5197.929 & Fe I (1091) & 0.0546 & -0.977 & $4.28-6.66$ & 0.583 & +31.18 \\
\hline 5200.874 & & UN & 0.0217 & & & & \\
\hline 5244.009 & 5243.306 & Fe III (113) & 0.0512 & 0.405 & $18.27-20.63$ & 0.703 & +37.75 \\
\hline 5262.238 & 5261.61 & {$[\mathrm{Fe} \mathrm{II}](19 \mathrm{~F})$} & 0.0375 & & & 0.628 & +33.34 \\
\hline 5273.955 & 5273.38 & {$[\mathrm{Fe} \mathrm{II}](18 \mathrm{~F})$} & 0.0182 & & & 0.575 & +30.24 \\
\hline 5282.946 & 5282.297 & Fe III (113) & 0.0350 & 0.108 & $18.27-20.61$ & 0.649 & +34.39 \\
\hline 5299.612 & 5299.045 & O I (26) & 0.0501 & -2.140 & $10.99-13.33$ & 0.567 & +29.63 \\
\hline 5343.07 & & UN & 0.0698 & & & & \\
\hline 5516.076 & 5515.335 & V I (1) & 0.0805 & -3.570 & $0.00-2.24$ & 0.741 & +37.84 \\
\hline 5535.998 & 5535.346 & V I (1) & 0.0293 & -4.043 & $0.02-2.25$ & 0.652 & +32.86 \\
\hline 5538.387 & 5537.760 & Mn I (4) & 0.0314 & -2.017 & $2.19-4.42$ & 0.627 & +31.50 \\
\hline 5555.575 & 5554.94 & O I (24) & 0.0327 & & $10.94-13.16$ & 0.635 & +31.82 \\
\hline 5577.325 & 5576.61 & Si II (9) & 0.0332 & & & 0.715 & +35.99 \\
\hline 5577.832 & 5577.35 & {$[\mathrm{O} \mathrm{I}](3 \mathrm{~F})$} & 0.0529 & & & 0.482 & +23.46 \\
\hline 5892.228 & 5891.598 & C II (5) & 0.1504 & -0.470 & $18.04-20.15$ & 0.63 & +29.61 \\
\hline 5920.922 & 5920.124 & Fe III (115) & 0.0634 & -0.034 & $18.79-20.88$ & 0.798 & +37.97 \\
\hline 5954.287 & 5953.613 & Fe III (115) & 0.0384 & 0.186 & $18.79-20.87$ & 0.674 & +31.49 \\
\hline 5958.201 & 5957.559 & Si II (4) & 0.1694 & -0.300 & $10.07-12.15$ & 0.642 & +29.86 \\
\hline \multirow[t]{2}{*}{5959.277} & 5958.46 & O I (23) & 0.0285 & & $10.94-13.01$ & & \\
\hline & +5958.63 & O I (23) & & & $10.94-13.01$ & & \\
\hline 5979.620 & 5978.930 & Si II (4) & 0.3998 & 0.000 & $10.07-12.15$ & 0.69 & +32.15 \\
\hline 6000.211 & 5999.543 & Fe III (117) & 0.0563 & 0.355 & $18.82-20.88$ & 0.668 & +30.93 \\
\hline 6033.247 & 6032.604 & Fe III (117) & 0.0830 & 0.497 & $18.82-20.87$ & 0.643 & +29.51 \\
\hline 6047.098 & 6046.46 & O I (22) & 0.0835 & & $10.94-12.98$ & 0.638 & +29.18 \\
\hline 6095.944 & 6095.37 & C II (24) & 0.0490 & & $22.47-24.50$ & 0.574 & +25.78 \\
\hline 6099.236 & 6098.62 & C II (24) & 0.0837 & & $22.47-24.50$ & 0.616 & +27.83 \\
\hline 6152.043 & & UN & 0.1118 & & & & \\
\hline 6244.028 & 6243.355 & Al II (10) & 0.0428 & 0.670 & $13.08-15.06$ & 0.673 & +29.87 \\
\hline 6257.864 & & UN & 0.0164 & & & & \\
\hline 6260.221 & & UN & 0.0242 & & & & \\
\hline 6300.877 & 6300.304 & {$[\mathrm{O} \mathrm{I}](1 \mathrm{~F})$} & 0.6924 & & & 0.573 & +24.81 \\
\hline 6347.803 & 6347.109 & Si II (2) & 0.3636 & 0.300 & $8.12-10.07$ & 0.694 & +30.33 \\
\hline 6364.348 & 6363.776 & {$[\mathrm{O} \mathrm{I}](1 \mathrm{~F})$} & 0.2262 & & & 0.572 & +24.49 \\
\hline 6372.111 & 6371.371 & Si II (2) & 0.1646 & 0.000 & $8.12-10.07$ & 0.74 & +32.37 \\
\hline 6462.585 & & UN & 0.1393 & & & & \\
\hline
\end{tabular}


Table 3b. continued.

\begin{tabular}{|c|c|c|c|c|c|c|c|}
\hline $\begin{array}{l}\lambda_{\text {obs. }} \\
(\AA)\end{array}$ & $\begin{array}{l}\lambda_{\text {lab. }} \\
(\AA)\end{array}$ & Ident. & $\begin{array}{l}W_{\lambda} \\
(\AA) \\
\end{array}$ & $\log (g f)$ & $\begin{array}{c}\chi \\
(\mathrm{eV})\end{array}$ & $\begin{array}{l}\Delta \lambda \\
(\AA)\end{array}$ & $\begin{array}{c}V_{\mathrm{r}} \\
\mathrm{km} \mathrm{s}^{-1}\end{array}$ \\
\hline 6546.69 & 6545.80 & Mg II (23) & 0.0218 & & $11.58-13.47$ & 0.89 & +38.32 \\
\hline \multirow[t]{2}{*}{6548.82} & 6548.1 & [N II] (1F) & 0.1552 & & & 0.72 & +30.52 \\
\hline & $6583.6^{\dagger}$ & [N II](1F) & & & & & \\
\hline 6611.462 & 6610.562 & N II (31) & 0.0364 & 0.430 & $21.60-23.48$ & 0.9 & +38.37 \\
\hline 6667.584 & 6666.938 & O II (85) & 0.0253 & -1.030 & $28.94-30.80$ & 0.646 & +26.60 \\
\hline 6731.683 & 6730.79 & C II (21) & 0.0412 & & $22.43-24.27$ & 0.893 & +37.33 \\
\hline 6751.248 & 6750.22 & C II (21) & 0.0191 & & $22.44-24.27$ & 1.028 & +43.22 \\
\hline 6794.664 & & UN & 0.0641 & & & & \\
\hline 6852.518 & & UN & 0.0321 & & & & \\
\hline \multirow[t]{2}{*}{7002.964} & 7001.93 & O I (21) & 0.0958 & & $10.94-12.70$ & & \\
\hline & +7002.22 & O I (21) & & & $10.94-12.70$ & & \\
\hline 7042.823 & 7042.048 & Al II (3) & 0.0695 & 0.350 & $11.32-13.08$ & 0.775 & +30.55 \\
\hline 7053.906 & 7052.9 & C II (26) & 0.0323 & & $22.80-24.55$ & 1.006 & +40.32 \\
\hline 7057.413 & 7056.612 & Al II (3) & 0.0512 & 0.130 & $11.32-13.07$ & 0.801 & +31.58 \\
\hline 7156.014 & 7155.14 & [Fe II] (14F) & 0.0320 & & & 0.874 & +34.17 \\
\hline 7232.065 & 7231.332 & C II (3) & 0.801 & 0.070 & $16.33-18.04$ & 0.733 & +27.94 \\
\hline \multirow[t]{2}{*}{7237.489} & 7236.421 & C II (3) & 1.072 & 0.330 & $16.33-18.04$ & & \\
\hline & +7236.91 & S II (18) & & & $14.09-15.80$ & & \\
\hline 7255.214 & 7254.448 & O I (20) & 0.119 & -1.100 & $10.99-12.70$ & 0.766 & +29.21 \\
\hline 7378.794 & & UN & 0.1842 & & & & \\
\hline 7412.59 & & Atmos. & 0.0637 & & & & \\
\hline 7443.104 & 7442.298 & N I (3) & 0.0369 & -0.450 & $10.33-11.99$ & 0.806 & +30.02 \\
\hline 7469.175 & 7468.312 & N I (3) & 0.0555 & -0.270 & $10.33-11.99$ & 0.863 & +32.20 \\
\hline 7750.613 & & Atmos. & 0.0195 & & & & \\
\hline 7877.897 & 7877.054 & Mg II (8) & 0.1891 & 0.390 & $10.00-11.57$ & 0.843 & +29.64 \\
\hline 7897.18 & 7896.367 & Mg II (8) & 0.3615 & 0.650 & $10.00-11.57$ & 0.813 & +28.42 \\
\hline 8000.947 & 8000.12 & {$[\mathrm{Cr}$ II] $(1 \mathrm{~F})$} & 0.0765 & & & 0.827 & +28.54 \\
\hline 8126.292 & 8125.50 & {$[\mathrm{Cr} \mathrm{II}](1 \mathrm{~F})$} & 0.0501 & & & 0.792 & +26.77 \\
\hline 8188.652 & $8187.95^{*}$ & N I (2) & 0.0384 & & $10.28-11.79$ & & \\
\hline 8214.932 & & UN & 0.0631 & & & & \\
\hline 8217.301 & 8216.28 & N I (2) & 0.0582 & & $10.29-11.79$ & 1.021 & +34.81 \\
\hline 8224.161 & 8223.07 & N I (2) & 0.0979 & & $10.29-11.79$ & 1.091 & +37.33 \\
\hline
\end{tabular}

$\dagger[\mathrm{N} \mathrm{II}](1 \mathrm{~F}) 6583.6 \AA$ is blended with the emission component of the C II(2) 6582.882 A P-Cygni profile.

$*$ the N I (2) 8187.95 emission line is affected by atmospheric absorption lines in the region. 
G. Sarkar et al.: Hot post-AGB stars: IRAS 13266-5551 and IRAS 17311-4924, Online Material $p 9$

\section{Appendix A: High resolution optical spectrum of IRAS 13266-5551 (CPD-55 5588)}
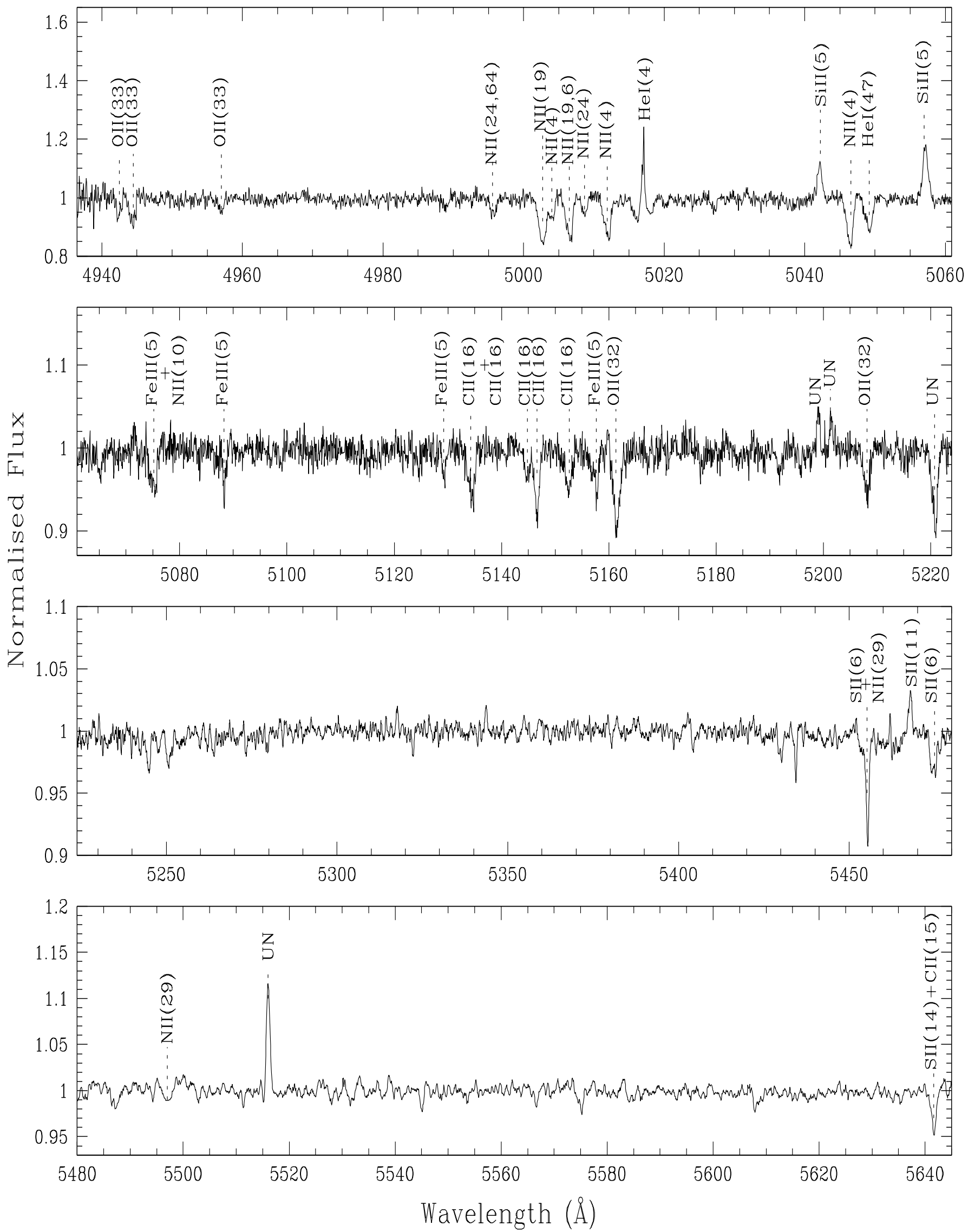

Fig. A. Optical spectrum of IRAS 13266-5551. 
G. Sarkar et al.: Hot post-AGB stars: IRAS 13266-5551 and IRAS 17311-4924, Online Material p 10

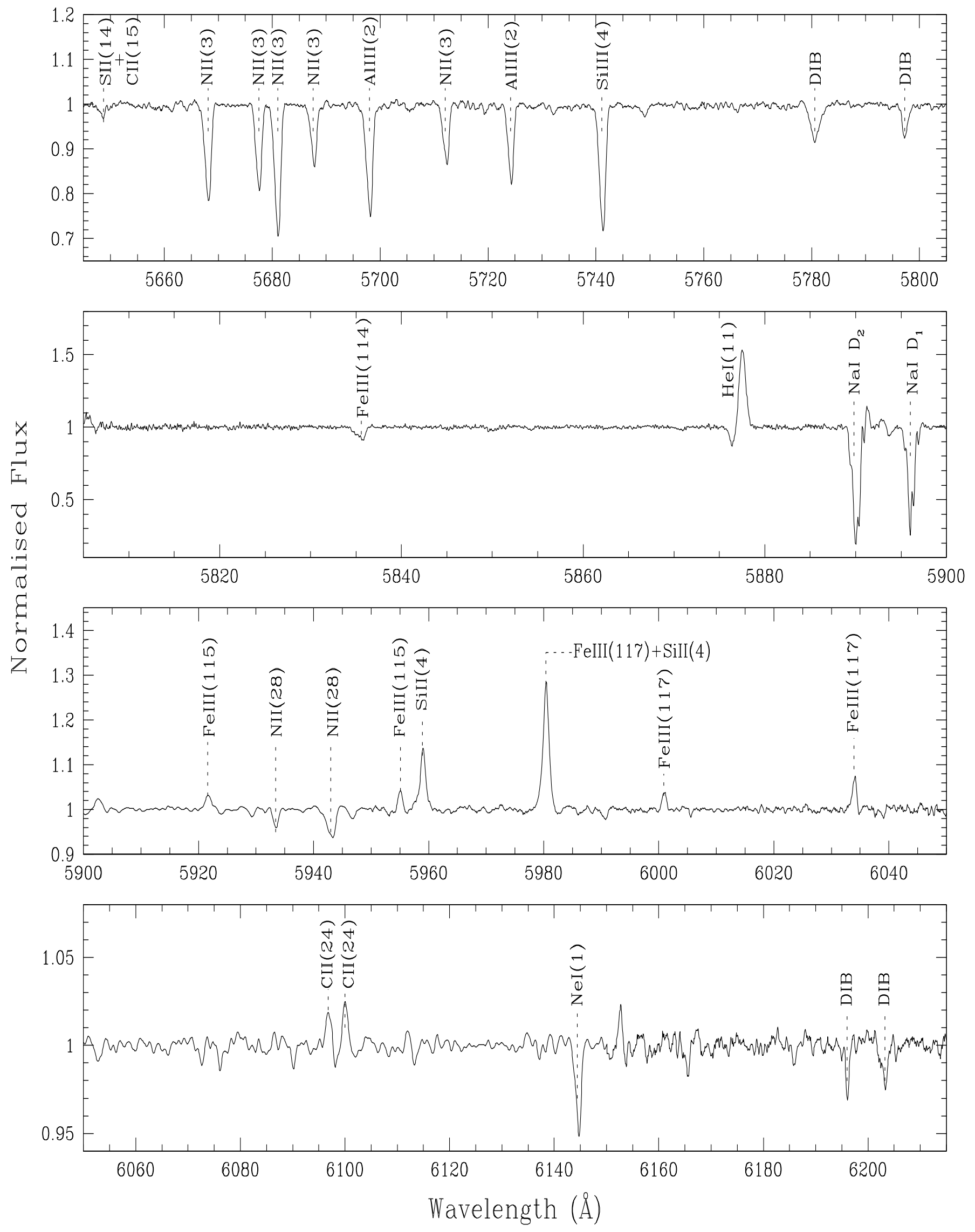

Fig. A. continued. 
G. Sarkar et al.: Hot post-AGB stars: IRAS 13266-5551 and IRAS 17311-4924, Online Material p 11

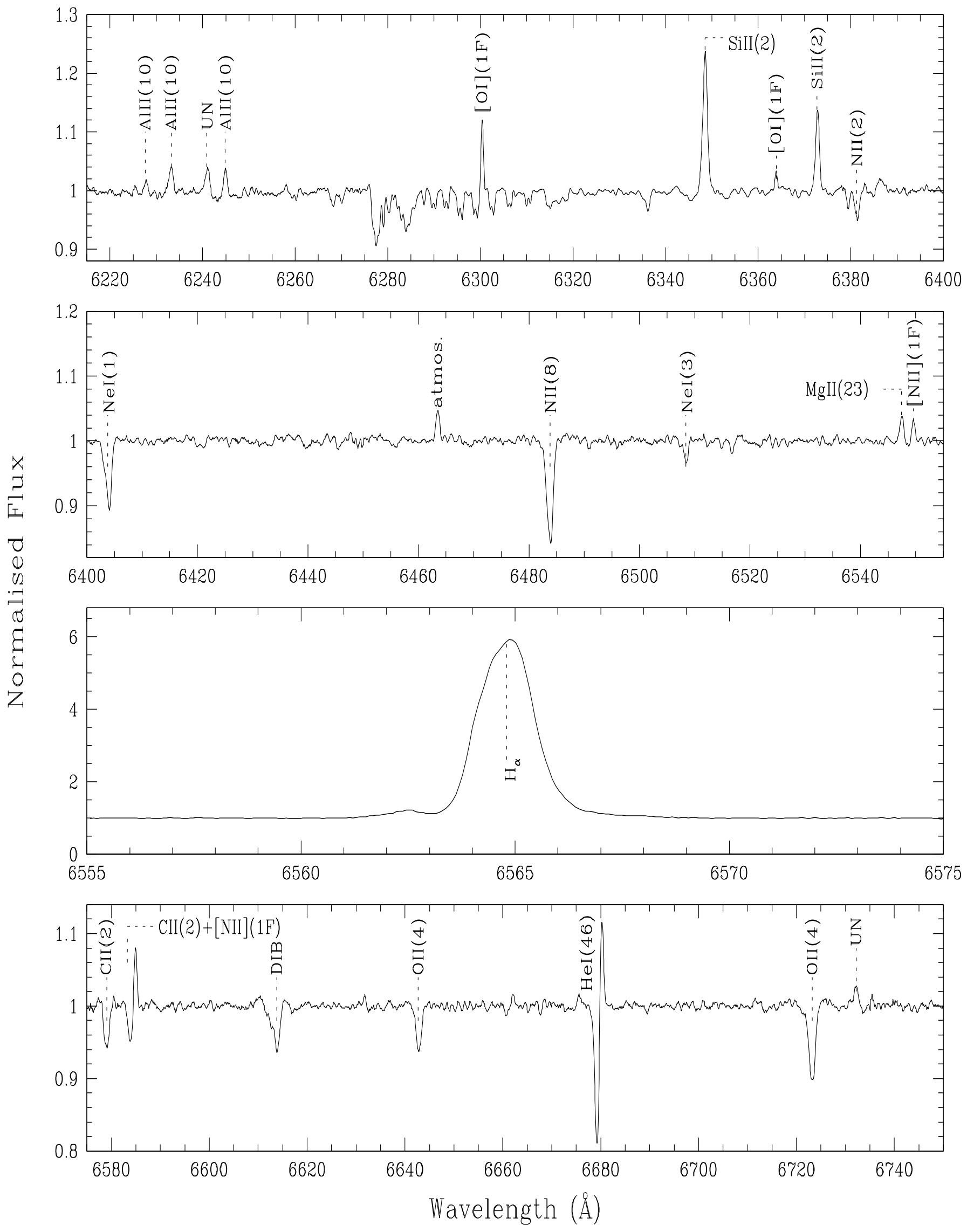

Fig. A. continued. 
G. Sarkar et al.: Hot post-AGB stars: IRAS 13266-5551 and IRAS 17311-4924, Online Material p 12
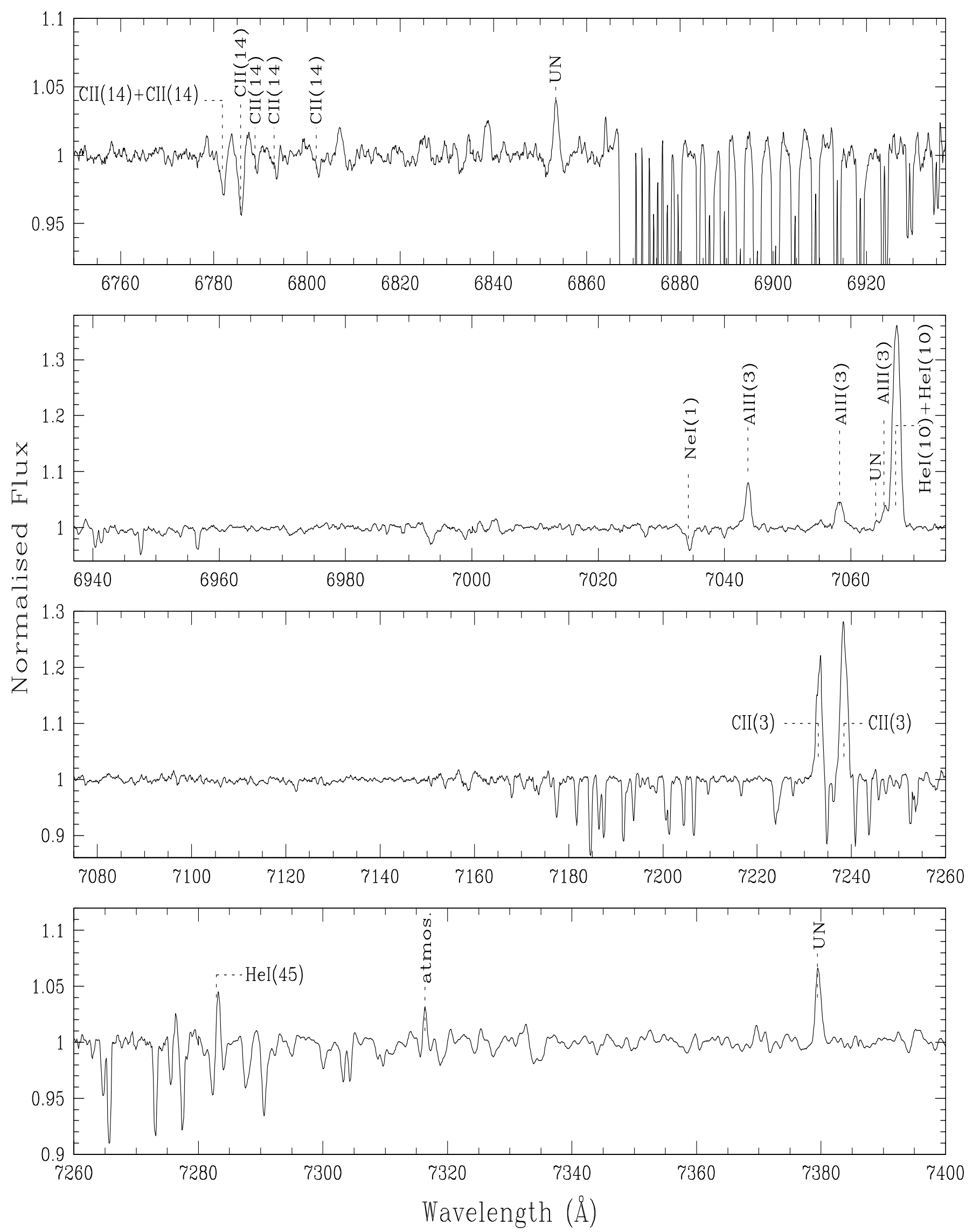

Fig. A. continued. 
G. Sarkar et al.: Hot post-AGB stars: IRAS 13266-5551 and IRAS 17311-4924, Online Material p 13
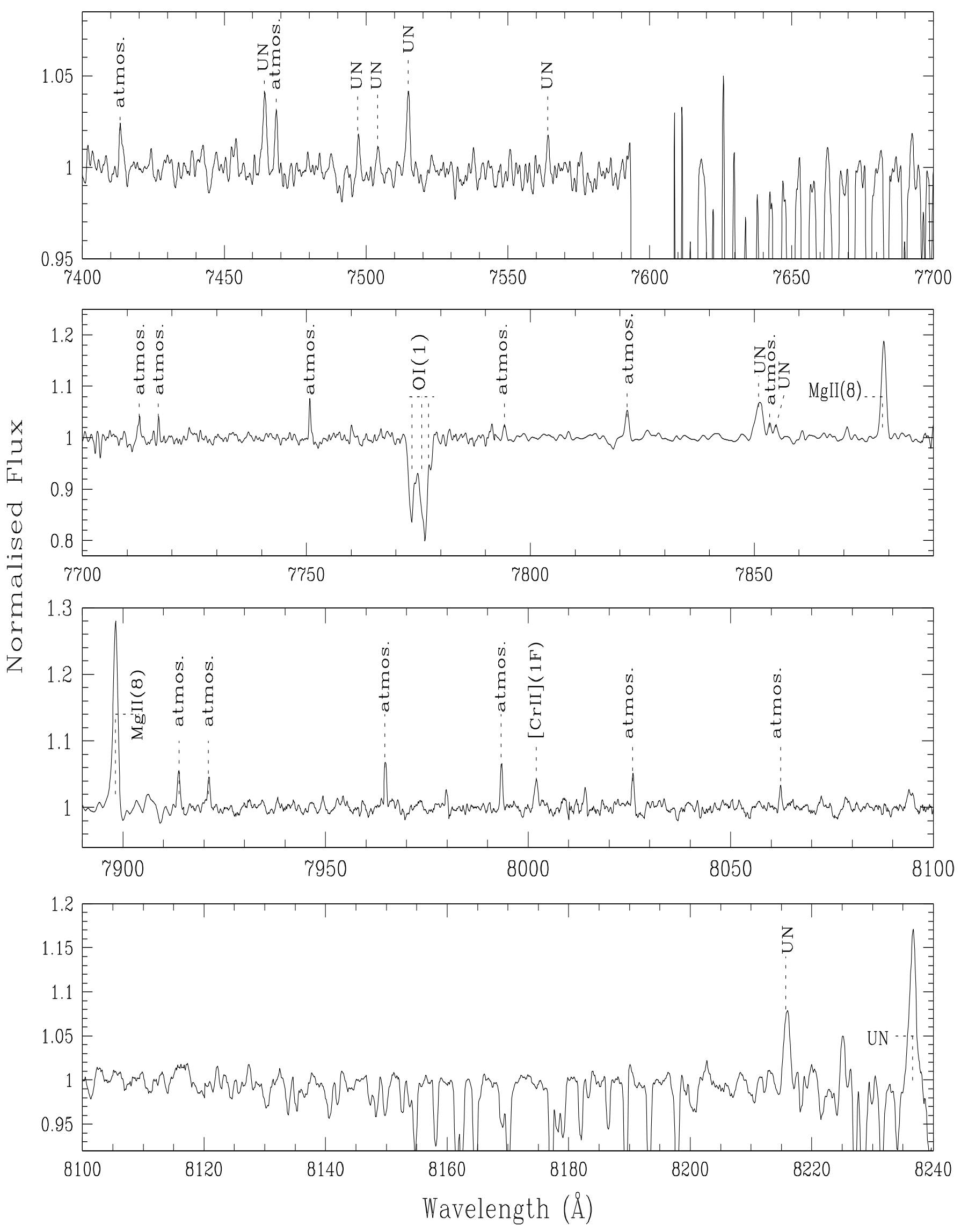

Fig. A. continued. 
G. Sarkar et al.: Hot post-AGB stars: IRAS 13266-5551 and IRAS 17311-4924, Online Material p 14

\section{Appendix B: High resolution optical spectrum of IRAS 17311-4924 (Hen3-1428)}

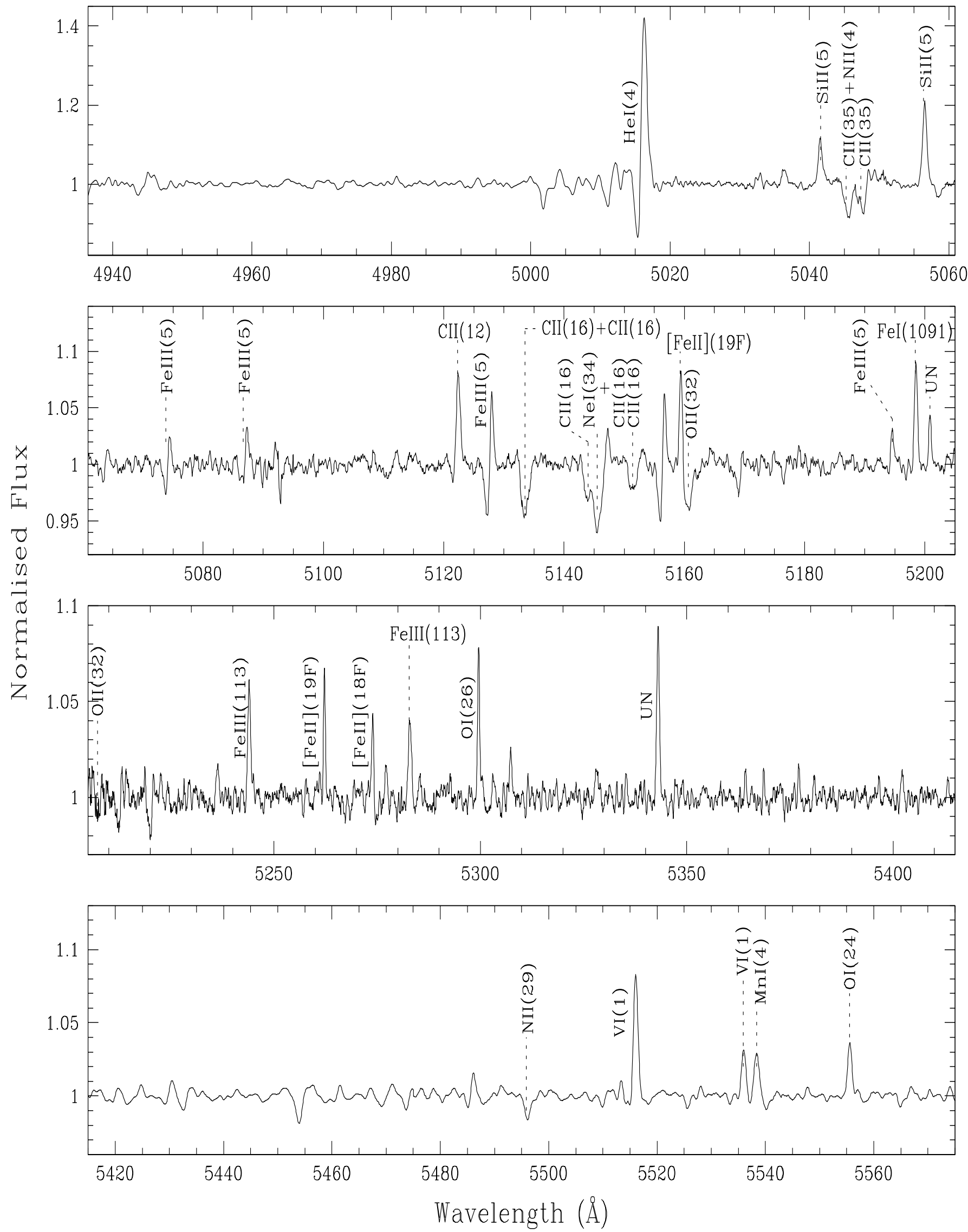

Fig. B. Optical spectrum of IRAS 17311-4924. 
G. Sarkar et al.: Hot post-AGB stars: IRAS 13266-5551 and IRAS 17311-4924, Online Material p 15
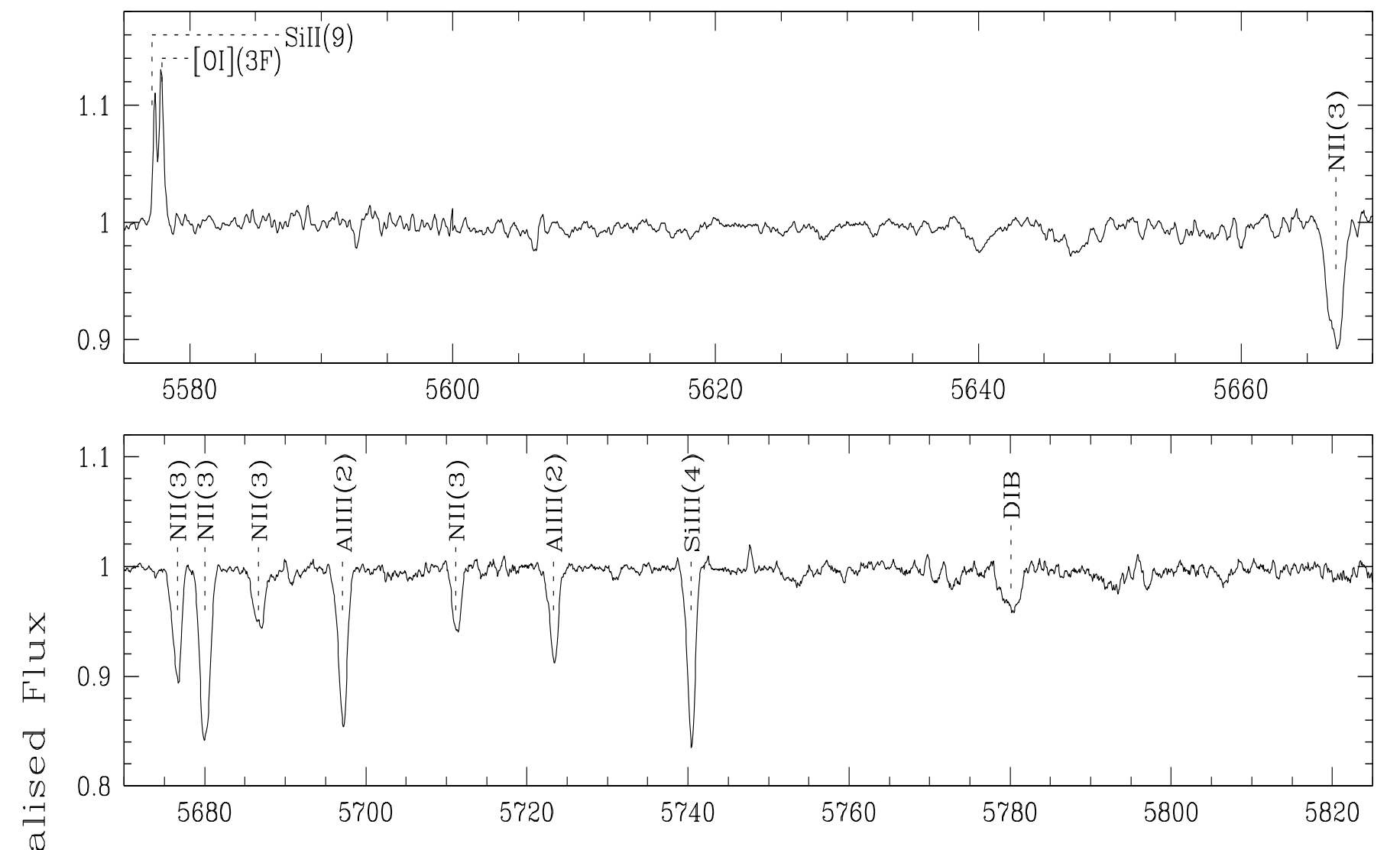

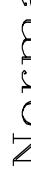
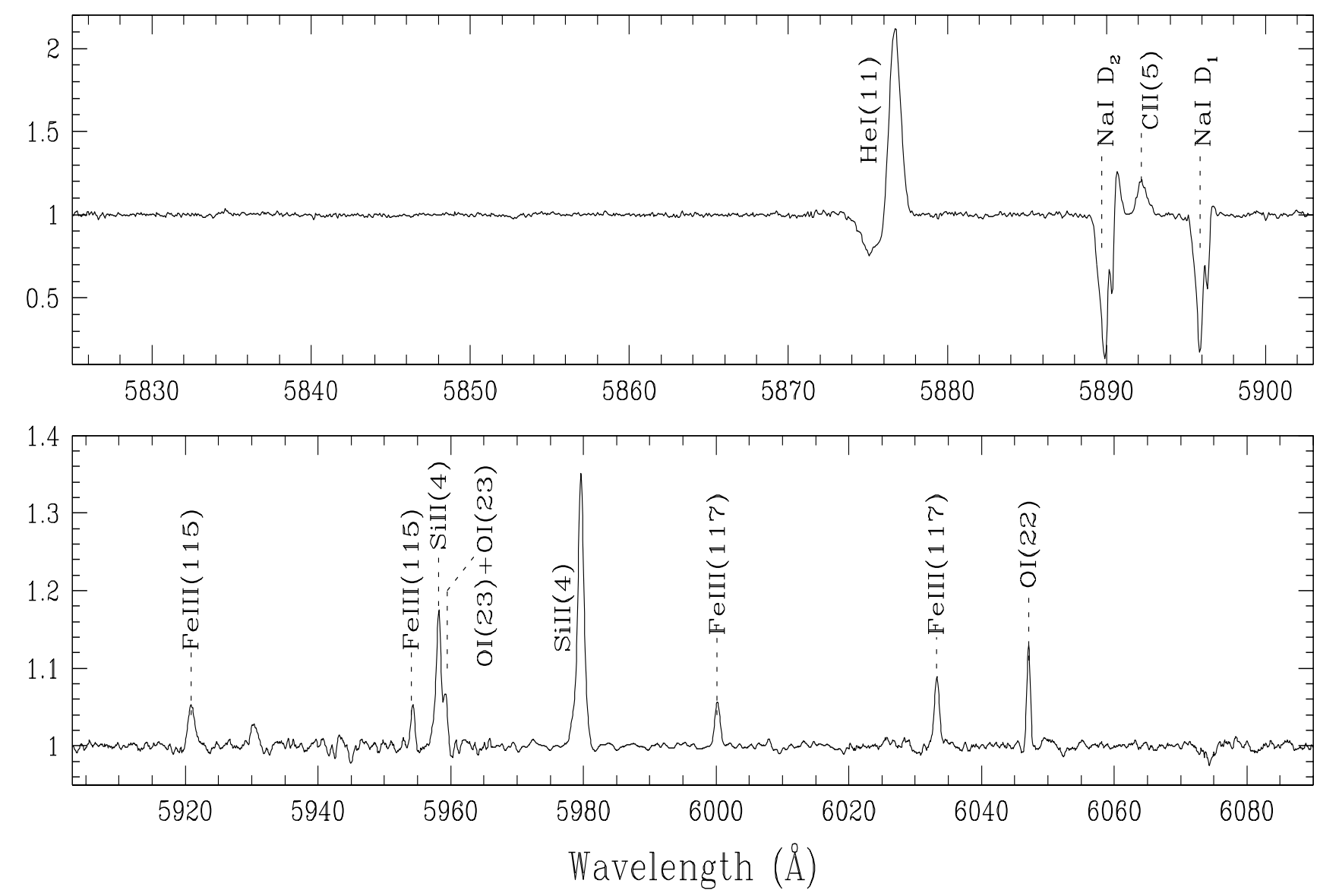

Fig. B. continued. 
G. Sarkar et al.: Hot post-AGB stars: IRAS 13266-5551 and IRAS 17311-4924, Online Material p 16
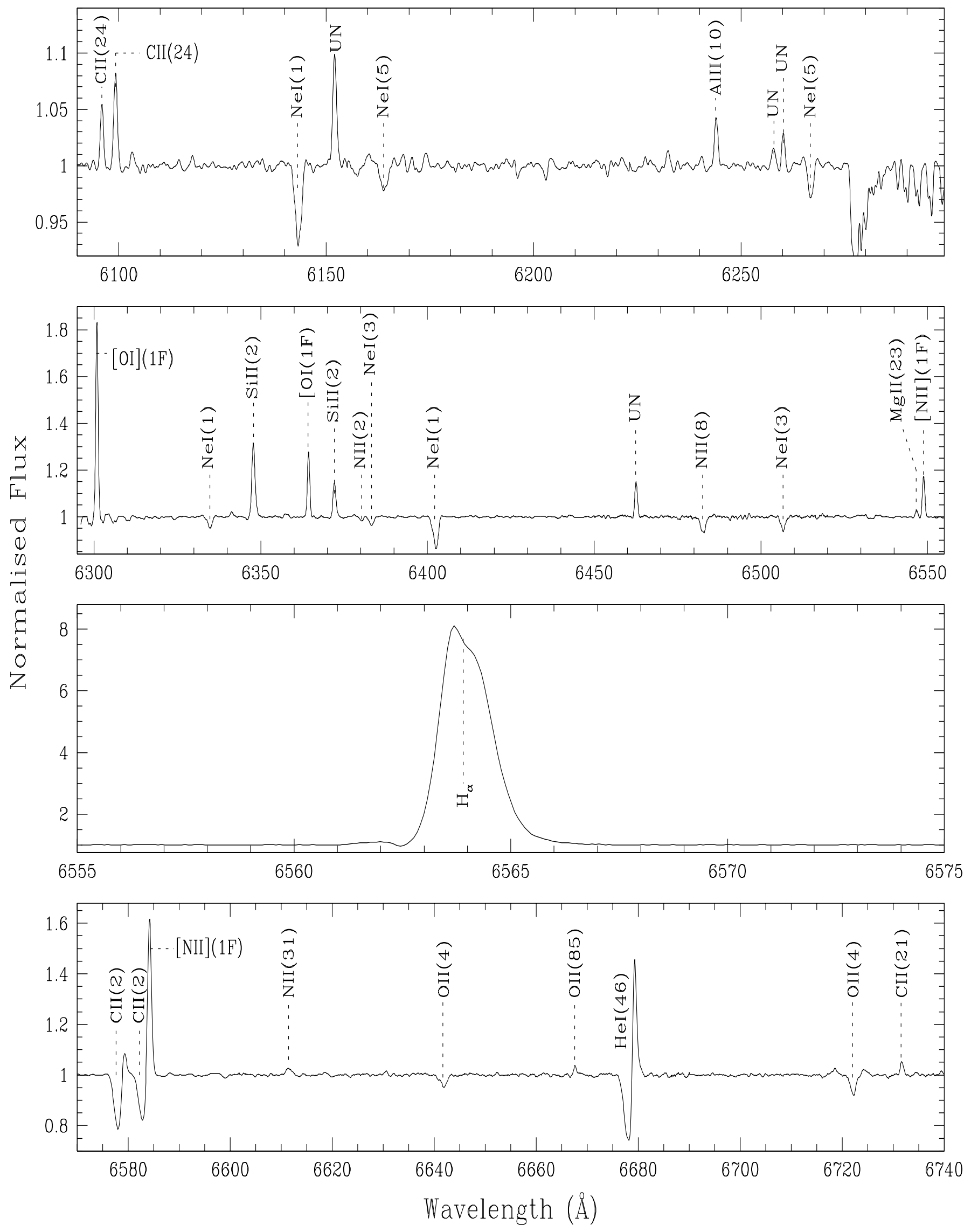

Fig. B. continued. 
G. Sarkar et al.: Hot post-AGB stars: IRAS 13266-5551 and IRAS 17311-4924, Online Material p 17
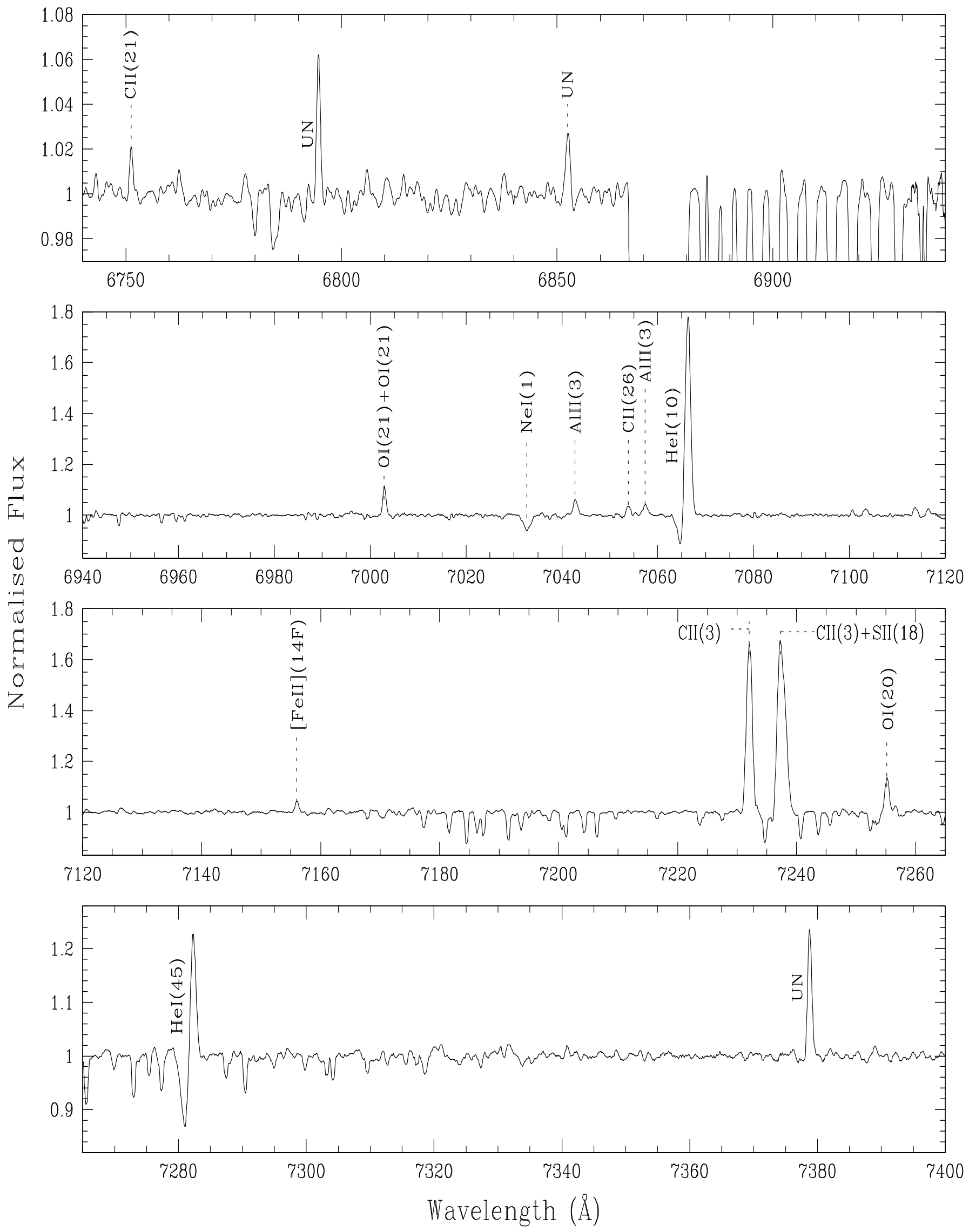

Fig. B. continued. 
G. Sarkar et al.: Hot post-AGB stars: IRAS 13266-5551 and IRAS 17311-4924, Online Material p 18

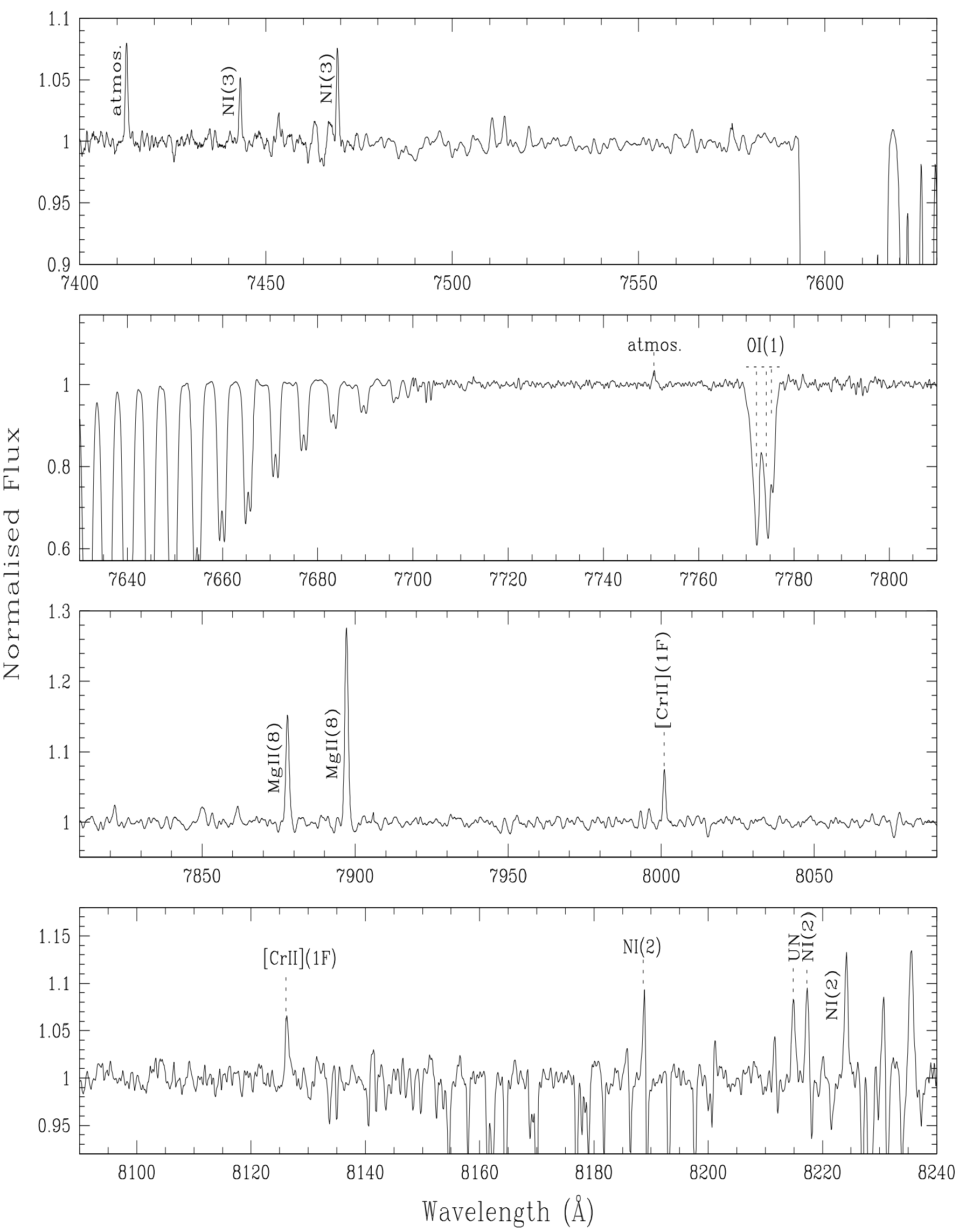

Fig. B. continued. 